\title{
Beitrag zur Kenntnis der Tuberkuloseverbreitung in Baden.
}

\author{
Von Dr. W. Hoffmann, \\ Assistent am patholog. Institut (Gel.-Rat A r nold).
}

\section{Verbreitungsweise der Infektionskrankheiten auf Grund ihrer ätiologischen Momente.}

Das Auftreten von Infektionskrankheiten geschieht innerhalb örtlicher und zeitlicher Grenzen. Nehmen wir die örtlichen Grenzen als Unterscheidungsmerkmal, so sprechen wir von epidemischen und endemischen Krankheiten einerseits, pandemischen andererseits; bevorzugen wir die Unterscheidung nach der Zeitdauer, so können wir von epidemischen und endemischen Krankheiten sprechen. Beide Gruppen werden gegeneinander sich nicht scharf abgrenzen lassen, da es unmöglich ist, eine Zeitdauer zu bestimmen, von der ab eine an einem Ort grassierende Krankheit als endemisch bezeichnet werden soll. Manche Krankheiten, z. B. Syphilis sehen wir innerhalb geschichtlicher Zeiten "epidemisch" auftreten, dann aber so fest sich einnisten, dass wir jetzt von "endemischem" Auftreten reden dürfen. Andere, wie z. B. Veitstanz oder schwarzer Tod, sind innerhalb geschichtlicher Zeiten aufgetreten, haben längere Zeit an einer Gegend gewütet, um wieder zu verschwinden. Das Gleiche gilt für Variola, nur ist hier die Ursache des Erlöschens in dem ärztlichen Eingreifen der Impfung zu suchen.

Im allgemeinen gilt der in seinen Folgerungen plausible Satz: Akute Infektionskrankheiten mit kurzem Verlauf zeigen meist epidemischen Charakter, ihr Verweilen am gleichen Ort ist kürzer, Infektionskrankheiten mit chronischem Verlauf mehr endemischen Charakter, ihr Verweilen am gleichen Ort ist länger. 
Die Frage nach dem Grunde des Einnistens von Krankheiten schung. in bestimmten Gegenden ist fast ebenso alt wie die medizinische Forschung (22). Je nach dem Stande der Erkenntnis wurden bald übernatürliche Kräfte, die Feindschaft einer Gottheit, oder ortsansässige dämonische Wesen, Hexen und Zauberer, bald Vergiftung der Brunnen, z. B. durch die Juden, bald aus dem Boden aufsteigende giftige Dämpfe, Miasmen u. a. m. in ziemlich kritikloser Weise beschuldigt. Dann mit dem Fortschritt der Wissenschaften wurden in klimatischen Finflüssen und in der Verbreitung organisierter Krankheitserreger die Krankheitsursachen gesucht und vielfach gefunden.

Ein Fehler geht jedoch heute den meisten derartigen pathogenetischen Forschungen nach. Es wird von vornherein vielfach nur einseitig nach einer einzigen Ursache ausgeschaut und geforscht, und ist dann die Möglichkeit eines Infektionsmodus gefunden, so wird derselbe als der einzige oder wenigstens als der verbreitetste bezeichnet. Durch diesen Fehler wird eine gewisse Einseitigkeit der Auffassung herbeigeführt, und in einem circulus vitiosus Gegner dieser Auffassung zu einer ebenso einseitigen Betonung ihres Standpunktes hingedrängt. Nur so ist oft eine Polemik von Meinungen erklärlich, die ganz gut nebeneinander bestehen könnten.

Es muss demgegenüber die Forderung erhoben werden, nur dann eine Form der Pathogenese als die einzig bestehende zu bezeichnen, wenn sie einerseits zwanglos alle Verbreitungserscheinungen erklärt, andrerseits nicht in Konkurrenz tritt mit anderen, ähnlich annehmbaren Erklärungsversuchen. Ist letzteres der Fall, so ist die Frage noch nicht spruchreif, oder es tritt die äusserst schwierige Aufgabe hervor, den prozentualen Anteil der verschiedenen Verbreitungsarten an dem Gesamtvorkommen zu bestimmen.

Die Ursachen für die natürliche Verbreitung der Krankheiten Momente. lassen sich in zwei grosse Gruppen einteilen: erstens solche, welche die Verbreitung der Krankheitserreger und die Infektionshäufigkeit beeinflussen, zweitens solche, welche den Menschen zur Aufnahme der Krankheitserreger geeigneter machen. Zu der ersten Gruppe gehören einerseits die Umstände, welche bestimmten Krankheitserregern das Leben nur an ganz bestimmten Orten gestatten, - als klassisches Beispiel, sämtliche Parasiten, welche bestimmte, nicht überall vorhandene Zwischenwirte zu ihrer Entwickelung nötig haben, - andrerseits die in den Lebensgewohnheiten der Menschen und ähnlichen Faktoren liegenden Ursachen für Verschleppung der Krankheitserreger und Ansteckung von Mensch zu Mensch. Eine viel mehr umstrittene Würdigung besitzt die zweite Gruppe, der Infek- die Ursachen, welche den Menschen zur Aufnahme der Krankheits-
tionsfahigkeit. 
erreger geeigneter machen. Von rein bakteriologischer Seite wird eine derartige Möglichkeit fast direkt in Abrede gestellt, die Mehrerkrankung bestimmter Menschenklassen und -Gruppen lediglich auf in diesen Gruppen und Klassen vermehrte Infektionshäufigkeit zurückgeführt. Nun hat aber das Tierexperiment gezeigt, dass selbst für den Injektionsversuch mit ihrer Virulenz und Zahl nach bekannten, gleichbleibenden Infektionserregern innerhalb der gleichen Tierspecies bedeutende quantitative Unterschiede der Empfänglichkeit sowohl, als nach erfolgter Infektion des Krankheitsverlaufs und der Krankheitsdauer bis zum letalen Ausgang bestehen. Es sind dies die Versuche von Wyss ok ow icz, Gärtner und Lubarsch, die letzterer(10) zusammenfassend verwertet, dann der Nachweis von Diphtherie-, Choleraund Typhusbacillen im Blute einzelner, nie an den betreffenden Krankheiten erkrankt gewesener oder erkrankender Menschen durch Wassermann, Abel, Klemperer, Stern; schliesslich die verschiedene extravaskuläre Vernichtungsfähigkeit des Blụtes Bacillen gegenüber, für Kaninchen und Milzbrandbacillen von $\mathrm{Nuttal}$, Nissen und Lubarsch festgestellt.

Man wird durch Analogieschlüsse aus derartigen Experimenten dazu gedrängt, auch bei den Krankheiten nach individuellen Verschiedenheiten der Reaktion auf die Krankheitserreger zu fahnden, bei denen ein experimenteller Nachweis einer "Disposition" des Individuums nicht in exakter Weise erbracht ist, oder aus technischen Gründen nicht erbracht werden kann. B a u m g a r t e n(10) suchte nun für die Tuberkulose den Gegenbeweis zu liefern und individuelle Schwankungen in der Disposition der Species auszuschliessen. Er glaubt feststellen zu können, dass die individuellen Schwankungen der Empfänglichkeit um so geringer sind, je hochgradiger empfänglich die betreffende Tierspecies ist. Als Massstab für die Empfänglichkeit stellt er die Häufigkeit der Spontanerkrankungen auf. Aus der Thatsache nun, dass der Mensch unter allen Tieren die höchste Erkrankungsziffer an spontanen Erkrankungen zeigt, will er den Schluss folgern, dass der Mensch zu den für Tuberkulose stärkst disponierten Tierspecies gehört, also auch die geringsten individuellen Schwankungen der Disposition zeige. In dieser Beweisführung ist vollkommen übersehen die Abhängigkeit der Erkrankungsziffern der verschiedenen Tierspecies von der für dieselben verschiedenen Infektionshäufigkeit. Für den Menschen sind die Infektionsgelegenheiten mit Tuberkulose weit häufiger als z. B. im Kaninchenstall, ein an Tuberkulose kranker Mensch, der etliche Jahre die Tuberkelbacillen weithin verbreitet, bedeutet eine viel grössere Gefahr als ein Kaninchen, das seine Miliartuberkulose abgeschlossen in sich birgt, selbst zur Infek- 
tion des Stalles nur wenig beiträgt, nach kurzer Krankheit derselben erliegt, wenn es nicht schon vorher als erkrankt erkannt und entfernt wird.

Weehselwirkung and indivi

dueller Mo
mente.

Es liegt also die Auffassung viel näher, nach dem Vorgange von Gottstein, Martius (11) und N a u s (14) das Zustandekommen von Infektionskrankheiten als auf einer Wechselwirkung zwischen dem befallenèn Organismus und dem Infektionsträger beruhend aufzufassen. Ob sich eine so streng formulierte, fast mathematische Gleichung, wie sie Gottstein vorschlägt, allgemeiner wird durchführen lassen, ist fraglich, doch wird der Standpunkt der Lehre von dieser Wechselwirkung in treffender Weise durch dies Verhältnis $\frac{W}{p}=$ Krankheitsursache zu Krankheitsanlage gekennzeichnet.

Es ergibt sich sich also als unabweisbare Forderung aus dem. Angeführten, bei pathogenetischen Studien Untersuchungen und Beobachtungen nach diesen beiden Richtungen anzustellen, Infektionsmöglichkeit und Häufigkeit einerseits, Verhalten des Individuums andererseits. Aus Kombination beider kann dann der Hygieniker Schlüsse auf die Verbreitungsweise der Krankheiten ziehen, und der innere Kliniker Beziehungen zu dem Krankheitsbild, der Erscheinungsform ableiten.

Patho- Betrachten wir von diesen Gesichtspunkten aus die Literatur Tuberkulose über die Pathogenese der Tuberkulose, so sollte man meinen, dass vorzugsweise diese Krankheit in ihren unzähligen Erseheinungsformen zur Vorsicht bei Aufstellung einseitiger pathogenetischer Theorien hätte mahnen sollen. Aber im Gegenteil, gerade bei dieser Krankheit, insbesondere ihrer häıfigsten Erscheinungsform, der Lungenschwindsucht, sind die Kontroversen der Meinungen am schärfsten, und der einseitig abgegrenzte Standpunkt der verschiedenen Forscher wird am hartnäckigsten verteidigt. Es stehen sich hier die reinen Infektionisten einiger bakteriologischen Schulen in ihren Hauptvertretern Baumgarten und Cornet (4-6) und die Nosoparasisten RiffeI (19-21) und Aufrecht (1) schroff gegenüber. Wohl die Mehrzahl der Ärzte nimmt eine bald mehr nach der einen, bald mehr nach der anderen Seite neigende Mittelstellung ein.

\section{Statistische Untersuchungsmethode.}

Es ist bei Anstellung klinischer Beobachtungen und Dentung des Ergebnisses des Tierexperimentes auf menschliche Verhältnisse häufig schwer, die subjektive Auffassung des Beobachters gänzlich zu vermeiden. Daher mag vielleicht noch mancher Widerspruch in der 
Literatur zu erklären sein. Den Vorteil grösserer Objektivität gewähren die Zahlen der Statistik. Zwar bedürfen auch sie einer Deutung, aber das System ihrer Zusammenstellung ist vollkommen klar und offen der Kritik zugänglich.

Es sei an dieser Stelle auf die durch Würzburg (33) präcis definierte AnfordeForderung der Statistik hingewiesen, die neuerdings Cornet wieder ${ }_{\text {selbe. }}^{\text {rongan }}$ scharf hervorgehoben hat, dass nur solche Zahlen vergleichbar sind und beweisenden Wert haben können, die sich als relative Werte zur Zahl der in der entsprechenden Gruppe Lebenden darstellen. Das in früheren und leider auch in etlichen neueren Statistiken vielfach als Mass der Mortalität verwandte Verhältnis der Mortalitätsziffer zu der Gesamtsterblichkeit kann nur in zweiter Linie und im Zusammenhang und Vergleich mit jenen anderen Zahlen eine Bedeutung behalten. Denn diese Verhältniszahl wird nicht nur von der Krankheit, als deren Mass sie aufgestellt ist, allein beeinflusst, sondern in gleicher Weise von der wechselnden Summe aller anderen Krankheiten, d. h. deren Mortalitätsziffern. Von grösserem Werte kann diese Zahl jedoch werden bei Vergleichung von Gruppen mit verschiedener Gesamtsterblichkeit, wo es von Interesse wird zu erfahren, wie viel von $\mathrm{Zu}-$ oder Abnahme auf Rechnung der einzelnen Krankheiten zu setzen ist.

Wenn wir nach dem Würzburg-Cornetschen Schema bei Berechnung der Tuberkulosemortalität nach Alter, Geschlecht u. dgl. verfahren müssen, so gilt natürlich das Gleiche bei Untersuchungen über Mortalitätsbestimmungen der Berufsarten, oder welche Gruppen man sonst in den Bereich der Untersuchung ziehen will. Soll z. B. für irgend ein Moment ein Einfluss auf die Tuberkulosemortalität auf statistischem Wege nachgewiesen werden, so ist der natürliche Weg der, festzustellen, dass in der gleichen Bevölkerungsgruppe sich dieses Moment bei den an Schwindsucht Erkrankten oder Gestorbenen häufiger findet als bei den nicht daran Erkrankten. Diese Feststellung ist aber nicht damit gelungen, wenn ein gewisser überwiegender Prozentsatz der an Phthise Gestorbenen dieses Moment darbietet. Es muss noch der Nachweis erfolgen, dass unter den an Phthise Gestorbenen dieser Prozentsatz grösser ist als unter der lebenden Bevölkerung oder Gruppen derselben. Ähnlich liegen die Verhältnisse bei dem Vergleich zweier geographischen Bezirke mit ihren Bevölkerungsgruppen. Es genügt nicht der Nachweis, dass bei an Schwindsucht Gestorbenen der einen Gruppe dieses Moment häufiger auftritt als bei der anderen. Der Ring der Beweisführung wird erst geschlossen durch Feststellung der gleichen oder verschiedenen Häufigkeit dieses Momentes bei den zu den beiden in Vergleich gezogenen Gruppen zugehörigen Lebenden. 
Ebenfalls von Cornet in gebührender Weise hervorgehoben ist die wichtige Tatsache, dass zur Aufstellung von Statistiken grosse Zahlen gehören, welche eine Unabhängigkeit von Zufälligkeiten verbürgen. „Die Statistik ist das Gesetz der grossen Zahl“, ruft Cornet mit voller Berechtigung denen $\mathrm{zu}$, welche aus keine 100 Fälle umfassendem Beobachtungsmaterial Prozentzahlen heraustüfteln und vergleichende Schlüsse damit ziehen wollen.

Eine andere äusserst wichtige Frage ist die: Lassen sich auf $\underset{\substack{\text { der } \\ \text { statistik. }}}{\text { statistischem Wege Schlüsse auf den Einfluss einer „Disposition“ des }}$ Individuums ableiten? Bei der gewöhnlichen Art der Aufstellung von Statistiken vermissen wir den Einfluss eines solchen individuellen Faktors, welcher der Disposition entspräche. Daraus jedoch den Schluss zu ziehen, dass eine Disposition überhaupt nicht vorhanden sei, ist nicht richtig. Nehmen wir als Beispiel die Cornetsche Statistik über die Mortalitätsfrequenz der verschiedenen Altersklassen. Die weiteste, allgemeinste und auch kritikloseste Fassung des Dispositionsbegriffes, oder vielmehr ein Missbrauch des Wortes Disposition, bedeutete lediglich eine verschiedene Wahrscheinlichkeit an Phthise zu erkranken. So sprach man von Berufs- und Altersdisposition. Dagegen wendet sich nun Cornet und zeigt in sehr plausibler Weise, dass man die Mortalitätsziffern an Tuberkulose für die verschiedenen Lebensalter in Parallele setzen kann zu den Infektionshäufigkeiten dieser Altersklassen. Er konnte es damit zu einer fast Beweiskraft erreichenden Wabrscheinlichkeit bringen, dass eine Verschiedenheit der Durchschnittsposition der Altersklassen - er nennt es eine Prädisposition - nicht besteht. Dass überhaupt keine Verschiedenheit der Disposition, d. h. der Wehrkraft der Individuen innerhalb der einzelnen Altersklassen bestände, ist damit noch lange nicht bewiesen. Denn bei einem annähernd gleichartig bleibenden Gemenge aus Disponierten und Nichtdisponierten innerhalb der verschiedenen Altersklassen ergäbe sich statistisch das gleiche Bild. Es reduziert sich die verschiedene Disposition einzelner Individuen bei Betrachtung grosser Zahlen auf die Durchschnittsdisposition der Gesamtheit. Es könnte also im einzelnen die Erkrankung des Individuums, seine Auswahl, durch seine Disposition bedingt sein, während die Zahl der Erkrankungen im statistischen Nachweis sich scheinbar wesentlich als eine Funktion der Infektionshäufigkeit darstellt.

Ist aber nicht die Annahme eines gleichbleibenden Gemisches aus Disponierten und Nichtdisponierten etwas Unmögliches? Man könnte hier den Einwand erheben, aus diesem Gemische müsste allmählich eine Auswahl der Disponierten stattfinden und der Rest dann geringere Sterblichkeit zeigen, also mit zunehmendem Alter eine Ab- 
nahme der Mortalitätsziffern zu verzeichnen sein. Das würde jedoch nur richtig sein, wenn es allein eine angeborene Disposition gäbe. Immerhin braucht wohl auch dann der Abfall der Mortalitätsziffern infolge der langsamen Auslese kein rapider zu sein. Denn eine Ubiquität der Infektionsmöglichkeiten können wir wohl nicht in dem Sinne annehmen, dass alle Augenblicke eine Infektion erfolgt, wenn auch wohl alle Menschen Gelegenheit haben, Zeit ihres Lebens etliche Infektionsmöglichkeiten durchzumachen. In den späteren Jahren wird aber die Zahl der angeboren Disponierten durch diejenigen vermehrt, welche ihre Disposition erst acquiriert haben oder einer Summation von Infektionen nicht mehr gewachsen sind. Es kompensiert also gewissermassen die zutretende Zahl derer mit erworbener Disposition den Abgang der angeboren Disponierten.

Es sind dies alles hypothetische Erörterungen, die unsere Unkenntnis über die zahlenmässige Häufigkeit der Infektionsmöglichkeiten und den Prozentsatz Disponierter nur in dürftigster Weise zu verhüllen suchen. Über die Arten und das Zustandekommen der „Disposition" soll an anderer Stelle ausführlich verhandelt werden. Hier sei über die Beziehungen von Infektionshänfigkeit und Disposition zur Phthisemortalität nur so viel betont, dass man als fast sicher annehmen darf, die Infektionshäufigkeit in proportionales Verhältnis zu setzen zur Mortalitätshäufigkeit.

Erst da, wo sich bei sonst gleichbleibenden Bedingungen der Infektionsverhältnisse und äusserer Schädlichkeiten gröbere Verschiedenheiten der Erkrankungs- und Todeszahlen finden, wird man zu den mehr individuellen Faktoren, der Disposition des Einzelnen, der Familie, oder bei Betrachtung grösserer Bezirke im Reibmayerschen (17) Sinne zu Dispositionsverschiedenheiten nach bestimmten Inzuchtsklassen, einer Stammes- und Rassendisposition greifen dürfen.

Dass auf dem Wege des statistischen Vergleiches sich wissenschaftlich interessante Beziehungen werden feststellen lassen, erschien mit aus dem Grunde wahrscheinlich, da die Statistik gewissermassen der zahlenmässige Ausdruck und sicher erbrachte Nachweis dessen ist, was besonders in den für die Dispositionslehre eintretenden Schriften als Erfahrung der Ärzte bezeichnet wird. Da sich diese Ansicht in einem gewissen steten Gegensatz zu den Lehren der Bakteriologen befindet, so wäre hier eine Gelegenheit geboten, dieser Ansicht, falls sie richtig ist, zum Ausdruck zu verhelfen.

Eine weitere Frage ist die, ob sich das Grossherzogtum Baden Vorzügeder zu derartigen Untersuchungen eignet. Baden besitzt in den Arbeiten $\begin{gathered}\text { badiseheu } \\ \text { Statistik. }\end{gathered}$ seines statistischen Landesamtes ein so wertvolles statistisches Material, dass nur wenige andere Staaten, was Genauigkeit der Durcharbei- 
tung betrifft, konkurrieren können. Was die Erfüllung der Anforderungen an statistische Arbeiten betrifft, so ist durch die über $1^{1 / 2}$ Millionen zählende Bevölkerung für die Benützung nicht zu kleiner Zahlen gesorgt. Bei einer Verfolgung der Tuberkulosemortalität und der übrigen in Betracht kommenden Verhältnisse über einen Zeitraum von 10 Jahren, und Vergleichung der daraus gewonnenen Durchschnittszahlen dürften wohl von Zufälligkeiten nur noch sehr wenig abhängige Daten gewonnen werden können.

Aber nicht nur eine grosse Zahl gehört zu einer verlässlichen Statistik, sondern auch ein gut beobachtetes und gleichmässig verarbeitetes Material. In diesem Punkt werden sich manche Unzulänglichkeiten herausstellen. An diesen muss dann eine peinliche Kritik einsetzen, um Massgaben für die Bewertung der Zahlen zu erhalten.

Einer der Hauptmängel ist die Unsicherheit über die Richtigkeit der in den Sterberegistern eingetragenen Diagnose. Dieser Fehler ist um so hochgradiger, je weniger die ärztliche Behandlung Platz greift. Die relativen Zahlen der unter ärztlicher Behandlung Gestorbenen sind nun für die verschiedenen Gegenden ungleich; es erfolgen nach der Statistik des Jahres 1895 Schwankungen von 50,6 bis $84,6 \%$ in den Amtsbezirken, während sich noch grössere Unterschiede von 90,5 bis zu $46,8 \%$ in den bezirksärztlichen Bezirken finden, wo noch eine Trennung in Stadt- und Landbezirke statt hat.

Es seien hier die Zahlen der unter ärztlicher Behandlung und mit ärztlicherseits gestellter Diagnose Gestorbenen in Prozentzahlen der Gesamtsterblichkeit für das Jahr 1895 wiedergegeben.

Die höchsten Zablen, mehr als $70 \%$ zeigen:

$\begin{array}{llll}\text { Freiburg Stadt } & 90,5 & \text { Heidelberg } & 78,8 \\ \text { Karlsruhe Stadt } & 90,0 & \text { Baden } & 78,0 \\ \text { Heidelberg Stadt } & 90,0 & \text { Emmendingen } & 75,0 \\ \text { Konstanz } & 84,6 & \text { Wertheim } & 74,7 \\ \text { Freiburg } & 83,6 & \text { Mannheim } & 74,1 \\ \text { Pforzheim Stadt } & 81,2 & \text { Donaueschingen } & 73,5 \\ \text { Lörrach } & 80,5 & \text { Ettenheim } & 71,8 \\ \text { Müllheim } & 80,3 & \text { Lahr } & 71,7 \\ \text { Staufen } & 79,5 & \text { Schönau } & 71,6 \\ \text { Mannheim Stadt } & 79,5 & \text { Bonndorf } & 71,4 \\ \text { Schopfheim } & 79,1 & \text { Kehl } & 70,7 \\ \text { Karlsruhe } & 78,9 & \text { Heidelberg Land } & 70,5\end{array}$

Die niedersten Ziffern, weniger als $60 \%$ zeigen:

$\begin{array}{llll}\text { Wolfach } & 59,3 & \text { Buchen } & 57,1 \\ \text { Stockach } & 59,2 & \text { Eberbach } & 56,7\end{array}$




$\begin{array}{llll}\text { Karlsruhe Land } & 59,2 & \text { Mosbach } & 55,7 \\ \text { Tauberbischofsheim } & 59,1 & \text { Ettlingen } & 55,5 \\ \text { St. Blasien } & 59,0 & \text { Wiesloch } & 53,3 \\ \text { Rastatt } & 59,0 & \text { Messkirch } & 50,6 \\ \text { Adelsheim } & 57,6 & \text { Pforzheim Land } & 46,8\end{array}$

Diesen Zahlen entsprechend wird man die Genauigkeit der amtlicherseits erhobenen Mortalitässtatistiken nach Todesursachen bewerten dürfen, falls der Durchschnitt der gesamten Bevölkerung des Amtsbezirks als Einheit der statistischen Beobachtung zu Grunde gelegt wird. Ein $\operatorname{gan} z$ anderes Bild wird aber entstehen, sowie die Mortalität für bestimmte Bevölkerungsgruppen, z. B. Altersklassen, bestimmt werden soll. Da ist nun in den meisten Landgegenden gebräuchlich, meist nur bei Erkrankung der Familienernährer, also der im besten Lebensalter stehenden Altersklassen, zum Arzte zu schicken, während die alten Leute und kleinen Kinder meist ohne ärztliche Konsultation den Verlauf der Erkrankung durchmachen und zum letalen Ausgang kommen. Es werden sich infolgedessen die diagnostischen Fehler im frühen Kindesalter und höheren Greisenalter vermehrt vorfinden.

Es ist weiter behauptet worden, dass eine zum letalen Ausgang führende Phthise auch für den Laien so sichere Erscheinungen biete, dass von den Fehlern der Totenschanerdiagnosen gerade bei dieser Krankheit Abstand genommen werden könnte. Die Richtigkeit dieser Folgerung muss entschieden beschränkt werden. Es kann ja wohl die Phthise als solche erkannt, and für mittlere Altersklassen, wo wenig andere zur Verwechslung kommende Krankheiten auftreten, mag man auch dem obigen Schluss eine gewisse Richtigkeit zugestehen, für höhere Altersklassen jedoch werden die chronischen, mit reichlichem Auswurf einhergehenden Emphysembronchitiden, vielfach sogar manche "zehrenden" Krebskachexien von Laienseite als Phthise gedeutet und infolge von Mangel ärztlicher Konsultation auch als solche im Sterberegister figurieren. Die amtlichen Angaben über die Tuberkulosemortalität für diese Altersklassen werden zu hoch ausfallen, und aus diesem doppelten Grunde die Fehlerquellen der Statistik für das höhere Alter grösser sein als für die sogenannten Blütejahre.

Es liegt mir fern, diese Fehlerquelle so hoch einschätzen zu wollen, dass man auf sie gestützt einen Angriff gegen die Cornetsche Beweisführung für die zunehmende Phthisefrequenz im höheren Alter unternehmen könnte; denn selbst bei Abstrichen von 30\%, einer Zahl, die sicherlich viel zu hoch gegriffen ist, behielten die Cornet schen Zahlen noch ihre volle Beweiskraft. Es sei dies vielmehr lediglich 
eine Klarlegung zum kritischen Verständnis später anzustellender statistischer Ausführungen.

Forderung $\quad$ Es ist ein sehr bedauerlicher Missstand, dass die statistischen bididatts--
statistik. Morbidität bleiben unsere Kenntnisse immer noch lückenhaft. Die Cornetsche Methode zur Bestimmung der Zahl der Kranken durch Multiplikation der Mortalitätszahl mit der durchschnittlichen Krankheitsdauer hat nur für die von ihm benützten, über weiteste Bezirke und grösste Zahlen sich erstreckenden Statistiken Gültigkeit und Berechtigung; für die in Baden in Betracht kommenden kleineren Bezirke würden so gefundene Zahlen nur ungenaue Schätzungswerte repräsentieren.

Über die Beziehungen von Morbidität zu Letalität, welche ihren Ausdruck in der Mortalität finden, könnten solche aus der Mortalität zurückberechnete Morbiditätszahlen keinen Ausschluss erteilen. Die Beobachtung von Schwankungen und Verschiedenheiten in diesen Beziehungen wäre jedoch von grösster Wichtigkeit. Sehen wir bei gleicher Morbidität in verschiedenen Gegenden verschiedene Mortalität - $\mathrm{Zu}$ - und Abwanderung als bekannt gegeben -, ist also die Zahl der zur Heilung kommenden Fälle in der einen Bevölkerung grösser, so konnte die Frage nach den Gründen dieser besseren Heilerfolge uns vielleicht über in der Bevölkerung selbst, ihrer Widerstandskraft liegende Verhältnisse Aufschluss geben.

Ausserdem würde eine Morbiditätsstatistik, wie Brau er (2) für die Cigarrenarbeiter der Rheinpfalz ausführt, in viel genauerer Weise die Berufe berücksichtigen können. Die Erkrankung findet in den meisten Fällen aus dem Beruf heraus statt, der letale Ausgang erfolgt häufiger nach Berufsaufgabe oder vielfach nach Berufswechsel. Eine Berufsstatistik der Verstorbenen würde also den Tatsachen nicht ganz entsprechende Bilder geben, ganz abgesehen von den Schwierigkeiten der technischen Ausführung, oft sogar der Unmöglichkeit der Unterscheidung zwischen den Berufstätigen selbst und ihren berufslosen oder anderweitig beschäftigten Angehörigen.

ortsanalyse. Es verdient an dieser Stelle die Frage eine Frörterung, ob überhaupt das durch die staatlicherseits vorgenommenen Erhebungen gelieferte statistische Material allen Anforderungen genügen kann. Zur Anstellung des statistischen Vergleichs wäre es am zweckmässigsten, solche geographisch begrenzte Bevölkerungsgruppen als Ausgangspunkt und Systemeinheit anzunehmen, welche unter sich die grösste Übereinstimmung besitzen, eine gewisse homogene Zusammensetzung aufweisen. Als eine solche natürliche Einheit ist abgesehen von grösseren Städten der einzelne Ort, Dorf oder Städtchen anzusehen. Für den 
Ort als Einheit wären die statistischen Ermittelungen anzustellen, aus mehreren Orten mit gemeinsamen gleichen Verhä]tnissen wieder grössere Gruppen zusammenzustellen. Der einzelne Ort ist das Objekt einer genauen analytischen Forschung über Schädlichkeiten des Klimas, der Beschäftigungsweise und Lebensweise der Bevölkerung, der Infektionsbedingungen und Häufigkeit derselben, eventuell der erblichen Dispositionsverhältnisse in den Familien. Es sind das Forderungen, wie sie an die amtlich aufgestellte Statistik unmöglich gestellt werden können.

Diese Verhältnisse müssen durch ad hoc angestellte Untersuchungen erst kennen gelernt und festgelegt werden, und durch sie die staatlichen Erhebungen ergänzt werden. R if fel (19-21) hat derartige Ortsanalysen als Untersuchungsmethode eingeführt, in seinen Arbeiten jedoch auf die Frmittelung der Infektionsbedingungen fast gar keinen Wert gelegt. Daher ist die scharfe Kritik zu verstehen, die seine Arbeiten von bakteriologischer Seite auszuhalten hatten.

Es tritt auch hier wieder eine Schwierigkeit hervor, die sich bei allen Statistiken fühlbar macht. Für einen bestimmten Zweck völlig ausnutzbar sind nur solche Statistiken, die für diesen Zweck angefertigt wurden, so dass schon die Sammlung des Materials nach diesem Gesichtspunkte erfolgen konnte. Diese leiden aber in mehr oder minder hohem Grade an den subjektiven Fehlerquellen des Verfassers, soweit sich solche bei Statistiken überhaupt bemerkbar machen können. Es muss daher das Bestreben vorhanden sein, durch Berücksichtigung möglichst vielseitiger und auch entgegengesetzter Momente diesen subjektiven Fehler möglichst auszugleichen und auszumerzen. Bei den nicht ad hoc verfertigten Statistiken bleiben häufig Momente infolge Nichtbeachtung auch nicht entdeckt; dadurch entstandene Fehler lassen sich nachträglich nicht mehr korrigieren und führen infolgedessen häufig zu gänzlich falschen Bildern.

Eine derart eingebende ortsanalytische Arbeit über grössere Bezirke ausgedehnt erfordert schon allein zur erstmaligen Feststellung der oben erwähnten Gesichtspunkte eine längere Arbeitszeit. Ob sich dann schon endgültige Schlüsse werden ziehen lassen, bleibt noch sehr fraglich. Denn viel länger als über ein Menschenalter wird sich die Untersuchung aus praktischen Gründen nicht erstrecken lassen. Ein Menschenalter ist aber für die Entwickelung der Tuberkulose, insbesondere bei Berücksichtigung der eventuell oder wahrscheinlich vorhandenen hereditären Momente ein äusserst kurzer Zeitraum. Man müsste vielleicht der einmaligen Feststellung für einen vergangenen bis zur Gegenwart reichenden Zeitabschnitt noch eine fortlaufende Beobachtung für die Zukunft folgen lassen. Dann aber kann man 
sich der Hoffnung hingeben, genauere Kenntnisse der Pathogenese zu erwerben, namentlich hinsichtlich der Unterscheidung zwischen Familieninfektion und hereditärer Disposition.

\section{Verbreitung der Schwindsucht in Baden.}

Einteilungs

Es soll nun versucht werden, geführt von den erwähnten Gesichtspunkten, zunächst ein Bild von der Verbreiturg der Tuberkulose in Baden zu geben. Als Ausgangspunkt für die statistischen Daten dienten die vorzüglich durchgearbeiteten Veröffentlichungen des statistischen Landesamtes für Baden: „Statistische Jahrbücher für Baden" und "Statistische Mitteilungen über das Grossherzogtum Baden", welch letztere einen besonderen, der medizinischen Statistik gewidmeten, umfangreichen Abschnitt enthalten. Nach dem früher Erwähnten ist aber die Feststellung von Beziehungen der geographischen und sozialen Verhältnisse nur als eine vorbereitende Arbeit gedacht, gewissermassen eine Rekognoszierung über Aufenthaltsort, Auftstellung und Taktik des Feindes.

Aus rein praktischen Gründen ist zur Erreichung dieses Zwecks als Einheit der Untersuchung der Amtsbezirk gewählt worden. Für eine natürliche Einteilungsweise sind zur Zeit noch keine fertigen statistischen Erhebungen vorhanden. Dass diese Einteilungsweise nach willkürlichen staatlichen Verwaltungsbezirken manche Missstände in sich birgt, ist von vornherein klar. Soweit sie analysierbar sind, können sie kritisiert, und die aus ihnen gezogenen Schlüsse entsprechend modifiziert werden. Es sind das namentlich Zustände, wo sich Amtsbezirke über verschiedene klimatische und geographische Lage erstrecken, wo Amtsbezirke ganz unter dem Einfluss einer, in ihnen oder im Nachbarbezirk liegenden grösseren Stadt sich befinden, wo Grenzen von Industriegebieten oder verschiedenen Volkstypen einen Amtsbezirk zerlegen. Da kann wohl manches der Kritik unterworfen werden, mehr jedoch wird vorläufig unerkannt bleiben, so dass manche Angaben cum grano salis zu nehmen sein werden. Das gilt insbesondere auch von Angaben über Durchschnittsbesitz, Einkommen, Ernährung, Alkoholkonsum und ähnliches. Näher auf die Bewertung des Zahlenmaterials im einzelnen wird an diesen verschiedenen Punkten eingegangen werden.

Es besteht ein gewisser Gegensatz in der Verbreitung der Tuberkulose auf dem Lande und den Städten. Derselbe macht sich besonders bei Vergleichen über Zu- und Abnahme der Krankheit bemerkbar. Es könnte nun mit Rücksicht hierauf ein Erfordernis zu sein scheinen, die Städte von den Landbezirken zu trennen. Bei der 
Durchführung einer solchen Spezialisierung treten aber Schwierigkeiten und Komplikationen auf, die in vielen Beziehungen hindernd und störend wirken können. Wenn z. B. bei den industriellen Berufen mit Wohnung der Arbeiter auf dem Lande und Arbeitsplatz in der Stadt, oder bei dem Zuzug der Kranken der Landbevölkerung zu den meist in den Städten befindlichen Krankenhäusern sich eine Trennung zwischen einheimischer Stadtbevölkerung und Zuzug aus der Umgebung wird durchführen lassen, dann haben die durch eingehende Untersuchung und Detaillierung bei Trennung von Stadt und Land gefundenen Zahlen ihre Berechtigung, und sind den die Amtsbezirke gesamt umfassenden bei weitem vorzuzieben. Eine solche Trennung ist aber fast nur bei ganz spezieller Ortskenntnis und auch da nur unter Zuziehung ausserordentlicher Hilfsmittel durchzuführen.

Im allgemeinen sind Land und die das industrielle Centrum darstellende Stadt so fest miteinander verbunden', es findet ein so lebhafter Austausch, ein so vielfaches Zusammentreffen der Bewohner beider statt, die Stadt bildet den Arbeitsplatz für die industriell thätige, den Verkaufs- und Kaufplatz für die landwirtschaftlich thätige Landbevölkerung, dass hier eine strikte Trennung, die nicht auf breiter natürlicher Basis begründet ist, oft sehr willkürlich einschneidend wäre. Es dürfte also, insbesondere zur vorläufigen Orientierung, die Einteilung nach Amtsbezirken gewisse Vorteile nicht verkennen lassen.

Wenden wir uns nun zu der Verbreitung von Krankheiten, so coographifindet sich in der medizinischen Statistik der "statistischen Mit- sche verteilungen über Baden fast stereotyp folgender Satz über das Verhältnis von Lungenschwindsucht und akuter Pneumonie- und Bronchopneumonie: "Die Sterbeziffern für Schwindsucht verhalten sich in den einzelnen Bezirken meist ähnlich, dagegen bietet die Sterblichkeit an Lungenentzündung häufigere und grössere Verschiedenheiten. Dabei ist der Landesdurchschnitt für beide Erkrankungen nur geringen Schwankungen ausgesetzt." Ähnliche nur noch stärkere Schwankungen wie die Langenentzündung zeigen auch die übrigen akuten Infektionskrankheiten. Gleichmässige örtliche Verbreitung und Schwankung nur innerhalb enger Grenzen in den verschiedenen Jahren zeigen vor allem zwei Krankheiten: Lungenschwindsucht und Krebs. Auf Tabelle I finden sich in Spalte 17 und 18 die Mortalitätsziffern dieser beiden Krankheiten berechnet für den Durchschnitt der Jahre 1891 bis 1900 auf je 1000 Lebende des Durchschnittes der drei Volkszählungen 1890, 95 und 1900.

Die niedrigste Mortalitätsziffer für Lungenschwindsucht zeigt BevölkeNeustadt mit $1,52 \%$ Todesfällen, die höchste Schwetzingen mit ${ }^{\text {rungsdichte. }}$ 
$4,26 \%$. Der Landesdurchschnitt liegt bei 2,84. Vergleichen wir mit dieser zahlenmässigen Darstellung die graphische Darstellung auf Tafel I, so fällt auf, dass der Schnittpunkt der den Landesdurchschnitt wiedergebenden Linie die ansteigende Linie der Tuberkulosehäufigkeit keineswegs in der Mitte ihrer Länge schneidet. Es ist dies der graphische Ausdruck dafür, dass die Mehrzahl der grösseren, volksreicheren Amtsbezirke sich auf der ungünstigeren Seite befindet, eine höhere Tuberkulosemortalität aufweist, als die kleineren oder dünner bevölkerten. Man könnte geneigt sein, darin direkt einen Einfluss der Volksdichtigkeit zu sehen. Es ist jedoch gewagt, die geographische Formulierung der Volksdichte auf das Quadratkilometer auf hygienische Verhältnisse übertragen zu wollen. Hier ist die Wohndichtigkeit von grösserem Einfluss. Allerdings ist es nicht leicht, für letztere einen zahlenmässigen Ausdruck zu verschaffen, da sehr viele Faktoren zu berïcksichtigen sind, Kubikraum der bewohnten Räume, Anzahl derselben, Anzahl der Häuser und Ausdehnung der Häuserkomplexe d. h. bebaute Bodenfläche und schliesslich noch Ausdehnung des Weichbildes. Zur Verwertung dieser Zahlen muss auch der praktischen Ausführbarkeit Rechnung getragen werden. Es ist in Baden eine Auszählung der bewohnten Räume im Gange. Nach deren Vollendung dürfte wohl durch die Verhältniszahl der Wohnräume zu der der Einwohner eine als Gradmesser der Wohndichte brauchbare Zahl gegeben sein. Einstweilen führten Vergleiche mit den von geographischer Seite $(28,15)$ erfolgten Arbeiten über Bevölkerungsdichte in Baden, insbesondere den kartographischen Darstellungen zu der Erkenntnis, dass sich Erwägungen, der sich einstweilen nur der geographischen Bevölkerungsdichtigkeit als Grundlage bedienten, allzusehr auf theoretisierendes Gebiet begeben; es werden infolgedessen diese besser unterbleiben. Als eine für uns später von Wichtigkeit werdende Tatsache wird jedoch von Uhlig (28) hervorgehoben, dass die zunehmende Bevölkerungsdichte ihren Grund in der Industriethätigkeit dieser Gegenden finden lässt.

Die Verbreitung der Tuberkulose von rein geographischem Standpunkt ans betrachtet, so findet sich ein grösseres Gebiet mit einer sich meist weit über Landesdurchschnitt erhebenden Tuberkulosemortalität in der unteren Rheinebene, den Amtsbezirken Baden, Rastatt, Ettlingen, Karlsruhe, Bruchsal, Wiesloch, Schwetzingen, Heidelberg, Mannheim, Weinheim und Eberbach entsprechend. In diesem Gebiete zeigt sich wieder eine komplexe Gruppe, Bruchsal, Wiesloch, Schwetzingen, Heidelberg, Eberbach mit einer Mortalität über $3,5 \%$, die in Schwetzingen mit einer Mortalität von $4,26 \%$ die ungünstigsten Verhältnisse aufweist. Die Erhebung der Mortali- 
tätsziffer im Amtsbezirk Baden auf $3,54 \%$ ist besonders auffällig, da sie wohl nicht auf die durch den Fremdenverkehr in der Stadt Baden herbeigeführten Verhältnisse zurückzuführen sein dürfte, da die Mortalität in dem Landbezirk grösser ist als in der Stadt selbst.

Im südlichen Teile der Rheinebene erheben sich nur die Bezirke Lahr, und dann die benachbarten Freiburg und Staufen über den Landesdurchschnitt, und auch deren Mortalitätszahlen, die bei Freiburg noch durch die Kliniken der Universität in ungünstiger Weise beeinflusst werden, erheben sich nicht zu der im nördlichen Baden beobachteten Höhe.

Günstige Verhältnisse zeigen die Bezirke des Odenwaldes, der Taubergegend und des Baulands, dann ein Komplex der Amtsbezirke Pforzheim, Bretten, Durlach. Schliesslich der ganze südliche Teil des Rheintals, der Schwarzwald, die Baar und die Seegegend. Die günstigsten Mortalitätsziffern unter 2\% zeigen die Amtsbezirke Buchen, Kehl, Neustadt, Bonndorf, Überlingen.

Vergleichen wir mit dieser Verbreitung der Tuberkulose diejenige des KrebsverKrebses = malignerTumor, die in gleicherW eise durch analoge Berechnung der Mortalitätszahlen festgestellt wurde, so ergibt sich ein fast direkt entgegengesetztes Bild. Im ganzen ist die Mortalitätsziffer niedriger als die der Schwindsucht, sie zeigt einen Landesdurchschnitt von 0,88\%, erreicht das Maximum mit 1,58 in den Amtsbezirken Stockach und Pfullendorf und das Minimum mit 0,59\% in Pforzheim. Eine stärkere Verbreitung durchweg über Landesdurchschnitt findet sich in den südlichen Amtsbezirken, die im oberen Rheintal und der Seegegend die ungünstigsten Verhältnisse, Mortalitätsziffern über 1,5\% bieten. In den nördlichen Amtsbezirken erheben sich nur in Heidelberg, Bruchsal, Weinheim und Eberbach die Mortalitätsziffern zu geringer Höhe über Landesdurchschnitt. Es fallen also die Gebiete höchster Tuberkulosemortalität mit denen niedrigster Krebssterblichkeit zusammen. Nur die Gebiete des Odenwalds, der Pforzheimer Gegend und Kehl zeigen gleichmässig niedrige Mortalitätszahlen fïr beide Krankheiten.

Aus dieser Art der Verbreitung dürfte wohl ersichtlich sein, dass ein innerer kausaler Zusammenhang, eine Schaffung einer besonderen Prädisposition für einander für die beiden Krankheiten nicht besteht, oder jedenfalls von anderen Ätiologiemomenten so sehr übertroffen wird, dass ein Einfluss auf die geographische Verbreitung nicht hervortritt. Jedenfalls kann aber die Riffelsche Behauptung, dass nicht nur in den von ihm untersuchten Familien - der Krebs häufiger in auch von der Tuberkulose angefallenen Familien sich finde, als widerlegt angenommen werden. Es ist damit keineswegs etwas 
gegen die Ansicht ausgesagt, dass nicht etwa ein Lupus die Ausgangsstelle bieten und Anlass geben könne zur späteren Entwickelung eines Carcinoms. Auch hier kann die Statistik nur für die grosse Zahl etwas aussagen, nicht für den einzelnen Fall. Das aber wird sicher gestellt, dass ein andere Momente übertreffender Einfluss nicht besteht, eher das Zusammentreffen beider Krankheiten bei ihrer Häufigkeit ein der mathematischen Wahrscheinlichkeit entsprechendes sein wird.

Um einen Zusammenhang der Tuberkulosemortalität mit Epidemien von Infektionskrankheiten nachzuweisen, müsste man auf die einzelnen Orte als Untersuchungseinheit zurückgreifen. Für den Amtsbezirk sind die Mortalitätszahlen an diesen anderen Krankheiten schon zu gleichmässig, als dass man die geringen Ausschläge irgendwie bewerten könnte. Aus den früher erwähnten Gründen ist ein solches Eingehen zur Zeit noch nicht möglich, würde auch an dieser Stelle zu weit führen.

Klimatische. Bei den grossen Differenzen, welche die klimatischen VerhältFinflüsse. nisse in Baden bieten, ist es interessant, Prüfungen anzustellen, in wieweit sich diese Verschiedenheiten in den Mortalitätsziffern der von uns verhandelten Krankheit zum Ausdruck bringen oder nicht.

In der Rheinebene, welche zu den wärmsten Gegenden Deutschlands gehört mit einem Jahresmittel der Luftwärme von $10^{\circ} \mathrm{C}$. und darüber, finden sich die Haupttuberkuloseherde. Auf der Hochebene der Baar, den Amtsbezirken Donaueschingen, Villingen etwa entsprechend, einer äusserst kalten und windigen Gegend, die ein Jahresmittel der Temperatur von nur $0,5^{\circ} \mathrm{C}$. aufweist und im Winter fast regelmässig Temperaturen unter $-25^{\circ} \mathrm{C}$. erreicht, finden sich sehr günstige Verhältnisse der Tuberkulosemortalität. Im Schwarzwald entspricht das Jahresmittel der Temperatur wohl der beträchtlichen Höhenlage, doch sind im Winter häufig warme Sonnentage, welche sogar ein Sitzen im Freien gestatten. In der Bodenseegegend macht sich der ausgleichende Einfluss der Wasserfläche auf die Temperatur geltend; es fehlt strenge Winterkälte und starke nächtliche Abkühlung. Die Tuberkulosenverhältnisse dieser beiden letzten Gegenden sind zwar recht günstig, aber um nichts besser als die in der rauhen und windigen Baar.

Von sonstigen klimatischen Einflüssen wäre vielleicht zu erwähnen, dass die Regenmenge in der nördlichen Rheinebene in der Umgebung von Mannheim am geringsten ist, am regenreicbsten der Westabhang des Schwarzwaldes, wie sich auch bei der Erwägung, dass die West- und Südwestwinde die feuchten Luftströme mit sich fuihren, als physikalisch notwendige Folge ergibt. Ob man berechtigt 
ist, aus diesen Thatsachen einen Einfluss auf die Tuberkulosenmortalität und ihre Verschiedenheit in den beiden Gebieten abzuleiten, erscheint mehr als zweifelhaft, doch sollten trotzdem die bestehenden Thatsachen ihre Erwähnung finden.

In höherem Masse verdient der Einfluss der Höhenlage Beachtung. Höhenłage. In der Literatur, welche sich mit diesem Thema befasst, kommt mehr der Einfluss der Höhenlage auf eine schon bestehende Phthise, also meist in Verbindung mit Luftwechsel u. s. w. zur Diskussion. Es handelt sich um die Frage nach einer spezifischen Wirkung der Hochgebirgskur. In seiner Tuberkulosestatistik für die Schweiz findet Schmid für die höheren Höhenlagen eine geringere Tuberkulosenmortalität als für die Niederung. Für Deutschland im ganzen kommt Köhle $\mathrm{r}$ zu keinen eindeutigen Resultaten. Geringere Höhenunterschiede, wie sie sich in den von Brauer untersuchten Cigarrenindustrieorten Nordbadens finden, scheinen überhaupt keinen Einfluss äussern zu können. Worin sich der Einfluss der Höhenlage äussert, ob lediglich infolge der dort häufigeren grossen Abgeschlossenheit rom Weltverkehr die Infektionsgelegenheiten seltener werden, das erscheint noch nicht sichergestellt. Die Verminderung des Staub- und damit Keimgehalts der Luft infolge der Luftverdünnung kommt wohl nur für die höchsten Erhebungen in Betracht. Es dürfte sich also immerhin lohnen, eine derartige Untersuchung für die mittleren Höhenunterschiede anzustellen, wie sie gerade in Baden vorkommen.

$\mathrm{Zu}$ diesem $\mathrm{Zweck}$ war es nötig, zunächst für die gewählte geographische Eimheit, den Amtsbezirk vergleichbare Höhenwerte zu finden. Eine geographische Durchschnittshöhe ist für unsere hygienische Zwecke nur äusserst mangelhaft verwendbar. Denn eine unbewohnte hohe Bergspitze in einem Gebiet ist hygienisch vollkommen gleichgiltig, während sie die geographische Durchschnittshöhe bedeutend in die Höhe treibt. Es dïrfen also nur die Höhen der bewohnten Orte zur Aufstellung des Durchschnittswertes verwandt werden. Geht man nun so vor, dass man die Summe der Höhen der einzelnen Orte bildet und durch die Zahl der Orte dividiert, so erhält man nur bei annähernd gleichmässiger Verteilung in den verschiedenen Höhen oder gleicher Einwohnerzahl richtige Durchschnittsergebnisse. Ist dies aber nicht der Fall, so könnte es z. B. vorkommen, dass in einer niedrig gelegenen Stadt der grösste Teil der Einwohner eines Bezirks zusammengedrängt ist, ausserdem aber noch eine grosse Anzahl hoch im Gebirge liegender ganz kleiner Flecken und Dörfer zu dem Bezirke gehören. Von diesen würde dann jedes auf die Durchschnittszahl den gleichen Einfluss ansüben wie die grosse Stadt, die Durchschnittszahl also viel zu hoch ausfallen. Es ist also nötig, den Einwohner- 
koeffizienten zu berücksichtigen und für den Einwohner die Durchschnittshöhe, in der er lebt, zu berechnen. Die Ausführung gestaltet sich also folgendermassen: Summierung der Produkte der Einwohnerzahlen mit Höhe des Ortes und Division durch die Gesamteinwohnerzahl des Bezirks.

Bei dem grossen Aufwand an Zeit, den eine solche Berechnung erfordert, wurde dieser Modus nur bei den Amtsbezirken durchgeführt, wo die einfachere Berechnung zu ungenaue Resultate bot, also den im Gebirge liegenden. Auf der Tabelle I sind ihre Namen durch ein Sternchen ausgezeichnet. Bei den in der Rheinebene liegenden ist diese Berechnưng unnötig, da die Differenzen der Höhe oft nur wenige Meter betragen. Die Details für die Orte der einzelnen Amtsbezirke hier wiederzugeben, wäre zu weitläufig; zur Technik der Ausführung sei nur bemerkt, dass die Höhenangaben der topographischen Karte im Massstab 1:25000 für das Grossherzogtum Baden entnommen sind, also die Gewähr grösstmöglicher Zuverlässigkeit bieten.

Die auf diese Weise gefundenen Höhenzahlen schwanken zwischen $866 \mathrm{~m}$ für St. Blasien und $100 \mathrm{~m}$ für Mannheim. Es liegen 16 Amtsbezirke mit 717992 Einwohnern, also nicht ganz die Hälfte der Einwohnerzahl in der Höhenlage zwischen 100-200 m. 13 Amtsbezirke mit 334437 Einwohner etwa ${ }^{1 / 5}$ der Einwohnerzahl in der Höhenlage zwischen 200 und $300 \mathrm{~m}$, zwölf Amtsbezirke mit 414312 Einwohnern, etwa 1/4 der Gesamteinwohnerzahl :zwischen 300 und 500 und elf Amtsbezirke mit 191206, also etwa $1 / 9$ der Einwohnerzahl in der Höhenlage über $500 \mathrm{~m}$. Die näheren Einzelheiten finden sich auf Tabelle II vermerkt. Ordnen wir nun die Amtsbezirke in der Reihenfolge ihrer steigenden Tuberkulosemortalität, wie es auf Tafel I geschehen ist und tragen die Höhen über dem Meeresspiegel als Ordinaten ein, so zeigt die Verbindungslinie der Endpunkte eine Zickzacklinie, welche im grossen und ganzen einen Abfall mit Zunahme der Mortalität erkennen lässt. Dieser Abfall der Tuberkulosemortalität wird noch deutlicher bei Betrachtung der verschiedenen Höhengruppen auf Tafel II. So zeigt die Höhengruppe I zwischen 100 und $200 \mathrm{~m}$ eine Durchschnittsmortalität von 3,18 auf 1000 Lebende, der Höhengruppe II zwischen 200 und $300 \mathrm{~m}$ eine solche von 2,56, der Höhengruppe III zwischen 300 und $500 \mathrm{~m}$ eine solche 2,58, die Höhengruppe IV zwischen $500 \mathrm{~m}$ eine solche von 2,06. Bei dieser Zusammenstellung ist zu bemerken, dass in Höhengruppe III der geringe Anstieg gegen II durch das Überwiegen der grossen Einwohnerzahlen mit verhältnismässig hoher Tuberkulosemortalität in Freiburg und Pforzheim bedingt ist. Die übrige Gruppe würde den erwarteten bedeutenden Abfall der Mortalitätsziffer in deutlichster Weise vorzeigen. Es wären 
dann, abzüglich Freiburg und Pforzheim, bei einer Einwohnerzahl von 273488 Menschen 2,35\% \%o Tuberkulosetodesfälle zu verzeichnen.

Eine Deutung dieser Zahlen ist an dieser Stelle noch verfrüht. Die Höhenlage ist gemeinschaftliche Ursache so mancher anderer Momente, welchen ihrerseits wieder ein Einfluss auf die Tuberkulosemortalität nicht abzusprechen ist, so dass erst in Zusammenhang mit diesen auch der reine Einfluss der Höhenlage als solcher diskutiert werden kann. Es wird vielleicht im folgenden gelingen einzelne Faktoren, welche in Konkurrenz mit dem Einfluss der Höhenlage treten oder einen denselben paralysierenden Einfluss haben können, wie z. B. gewisse Industrieverbreitungen aus dem allgemeinen, in sich unabgegrenzten Gemenge von wechselnden Einflüssen zu eliminieren. Aus der vorliegenden Aufstellung scheint jedoch mit Sicherheit so viel hervorzugehen, dass die Höhenlage in Baden Zustände schafft, welche der Verbreitung der Tuberkulose entgegenwirken. Es zeigen sich hier ähnliche Verhältnisse, vielleicht nur durch die verschiedene Art der Zusammenstellung noch deutlicher hervorgehoben, wie sie Schmid (23) für die Schweiz nachweist. Eine gewisse Skepsis bleibt jedoch noch immer angezeigt und vorläufig hat K ri e ger (8) die Beantwortung dieser Fragen dahin präcisiert, dass sie sich noch nicht aus dem Rahmen der Hypothese bewegt, und daran kann auch das Beibringen weiterer Tatsachen von geringerer Tuberkulosemortalität in grösserer Höhe ohne genaueste Analyse anderer mitwirkender Momente einstweilen nichts ändern.

\section{Einfiluss sozialer Verhältnisse und der Ernährungsweise.}

Ein bedeutender Einfluss wurde der Volksmeinung nach von jeher Armı drückenden sozialen Verhältnissen, Armut, Sorgen, Kummer, Entbehrung zugesprochen. Es sind ja nun vielfach mit der Armut $\mathrm{Zu}$ stände verbunden, welche eine Verbreitung der Schwindsucht plausibel machen, wie enges Zusammenwohnen und vielfach auch Unreinlichkeit. So bezeichnet $\mathrm{R}$ anso me (16) die Tuberkulose direkt also eine ,filthdisease". Es entbehrt nun eines gewissen Interesses nicht, den Versuch anzustellen, ob sich ein Zusammenhang feststellen lässt zwischen der Höhe der Tuberkulosemortalität und Zahlen, welche einen Massstab für die erwähnten Faktoren abgeben können.

Als eine die pekuniäre Leistungsfähigkeit der Amtsbezirke charakterisierende Zahl wurde aufgestellt die Durchschnittszahl des steuerbaren Einkommens pro Kopf der Bevölkerung. Die Zahlen sind ja wohl nicht ganz gleichmässig entsprechend der verschiedenen 
Zusammensetzung der Bevölkerung aus Reichen und Armen in den verschiedenen Bezirken, es lässt sich jedoch immerhin ein der Wirklichkeit entsprechendes Bild konstruieren. Genauere Zahlen sind schwer zu erhalten. Man könnte vielleicht so vorgehen, den Prozentbestand der Bevölkerung aus den verschiedenen Steuerklassen, insbesondere der niedersten, zu bestimmen. Wo diese Zahlen leicht zugänglich sind, wäre diese Methode wohl vorzuziehen; im einzelnen muss man aber den praktischen Verhältnissen Rechnung tragen und versuchen, das Vorhandene entsprechend zu verwerten.

Die Berechnung erfolgte in der Weise, dass aus den Angaben der Steuerverwaltung für die Jahre 1892, 96 und 98 das Mittel auf die Durchschnittseinwobnerzahl 1891-1900 berechnet wurde. Die auf diese Weise gefundenen Zahlen schwanken zwischen 1517 Mark im Amtsbezirk Eberbach und 4556 Mark im Amtsbezirk Mannheim. Das Landesmittel liegt bei 2340 Mark. Deutung dieser Zahlen und Folgerungen aus ihnen sind mit grösster Vorsicht anzustellen. Verfolgen wir die Kurve auf Tafel I, welche diese Zahlen graphisch darstellt, so sehen wir eine unregelmässige Zickzacklinie, der auch nicht der geringste Zusammenhang mit der die Tuberkulosesterblichkeit wiedergebenden Linie nachgewiesen werden kann. Die grösseren Erhebungen finden sich in der letzten Hälfte der Kurve bei den grossen Handels und Industriestädten, wo auch die Tuberkulose beträchtliche Höhe zeigt. Nun besteht aber gerade in diesen Bezirken ein Gegensatz zwischen Stadt- und Landbezirk derart, dass auf dem Lande die Tuberkulose grössere Verbreitung besitzt, als in der Stadt, wo sich der Grossbesitz findet. Es wäre also schon deswegen statistisch unmöglich, die höhere Tuberkulosemortalität mit dem grösseren Besitz. in Beziehung bringen zu wollen, abgesehen davon, dass dies allen anderen Erfahrungen direkt widersprechen würde. Wir müssen offen gestehen, dass wir auf diese Methode einen Zusammenhang nicht nachweisen können. Dass ein solcher Zusammenhang, falls er doch bestehen sollte, nur äusserst mittelbar sein kann, dafür spricht auch die von Virch ow (29) bei Gelegenheit des Hungertyphus in Schlesien gemachte Erfahrung, dass trotz der grössten Entbehrungen, welchen die dortige Bevölkerung eigentlich zeitlebens unterworfen war, keine nennenswerte Tuberkulosehäufigkeit zu beobachten war.

Andererseits ist auch die Höhe des Verdienstes, die sich in den angefuhrten Zahlen widerspiegelt, durchaus nicht immer gleichbedeutend mit den Mitteln, die in rationeller Weise zu vernüftiger Lebensführung verwandt werden. Fs spielen hier die mannigfachsten Gründe mit. Zunächst kommt in Betracht, wie viel dieses Verdienstes thatsächlich zur Ernährung und Wohnung verwandt wird. Von seiten 
der badischen Fabrikinspektion hat $\mathrm{W}$ ö $\mathrm{r} r$ is h o f er $(30)$ speziell für die Tabakarbeiter genaue Erhebungen über dieses Jahresbudget der Arbeiterfamilien ausgeführt.

Abgesehen von der geringen Höhe der überhaupt zu Gebote stehenden Geldmittel, die vielfach zum Schuldenmachen nötigen, zeigt sich ein Missverhältnis der Kosten für Genussmittel Bier und Kaffee, für welche oft mehr, fast überall aber die gleiche Summe aufgewandt wird, als für Brod und Fleisch. Die Zahlen schwanken zwischen $1 / 6$ und $1 / 10$ des Gesamtaufwandes für den Haushalt. Dabei ist das von dem Manne konsumierte Bier wohl vielfach der Berechnung entzogen. Ausserdem lässt sich feststellen, dass ein beträchtlicher Teil der Arbeiterbevölkerung in Unterernährung lebt, und diese bei den Frauen meist beträchtlicher ist als bei den Männern, die in egoistischerer Weise sich manche ausserordentlichen Zuwendungen gestatten.

Eine andere Ursache für die Ungleichmässigkeit der Beziehungen zwischen Einkommen und Umsatz desselben in geeignete Nahrungsmittel liegt in der vielfach beobachteten und beklagten Unfähigkeit der Arbeiterfrauen, mit den ihnen zu Gebote stehenden Mitteln eine geeignete Nahrung zu bereiten, so dass schliesslich das, was zur Aufnahme kommt, nicht im Verhältnis steht zu den dafür aufgewandten Mitteln. Bei der Frage nach den Gründen dieser Zustände kommen wir schon bedeutend auf sozialökonomisches Gebiet. Eine ausfiuhrlichere Behandlung an dieser Stelle dürfte sich wohl deshalb verbieten. Die Fragen sind jedoch zu interessant, als dass sich eine vollständige Übergehung derselben rechtfertigen liesse. Es wurde mir von seiten des badischen Ministeriums des Innern in dankenswertester Weise das Ergebnis einer diese Punkte betreffenden Umfrage zur Verfügung gestellt.

Mit Rücksicht auf die staatlicherseits einzuschlagenden Mittel zur Bekämpfung der Tuberkulose als Volkskrankheit war von seiten des Ministeriums an die badischen Bezirksärzte eine Umfrage gerichtet worden mit Charakterisierung folgender Punkte:

1. Ist in der Beschaffenheit der Wohnungen und in der Art der Ernährung ein die Verbreitung der Tuberkulose begïnstigender Faktor zu erblicken?

2. Wird bei den Kindern häufig Rhachitis und Skrofulose beobachtet?

3. Liegt im Falle ungenügender Ernährung der Grund hierzu lediglich in der Armut der betreffenden Familien oder in der Unkenntnis der Frauen mit Bezug auf die Zubereitung der täglichen Kost? 
4. Wäre durch weitere Verbreitung des Haushaltungsunterrichtes für Fortbildungsschülerinnen diesem Umstand vielleicht Rechnung zu tragen?

5. Bewirkt die Cigarrenfabrikation eine weitere Verbreitung der Ansteckungsgefahr und der Krankheit selbst?

Wird bei den Kindern von Cigarrenarbeitern häufiger als bei anderen Skrofulose und Rhachitis beobachtet?

In der Beantwortung dieser Fragen findet sich mit Ausnahme von Staufen und Baden fast überall das gleiche Klagelied über enge, überbelegte, zum Teil unreinliche Wohnungen, vielfach in so drastischer Darstellung, dass hier einiges im Original wiedergegeben sei. So schreibt Behrle von Schwetzingen: Ein Zimmer mit mehr als sechs, oder zwei Zimmer mit mehr als zwölf Personen belegt keine Seltenheit, gemeinsame Benutzung eines Bettes von bis zu vier Personen beiderlei Geschlechts. In Bruchsal nennt Klehe die Verhältnisse sehr günstig, wenn Wohnzimmer und Schlafzimmer mit wirklicher Zwischenwand und Küche der Familie zur Verfügung steht. Meist jedoch nur Trennung durch Schränke oder Vorhänge. Ähnlich läuten die Berichte aus den anderen Bezirken mit sehr hoher Tuberkulosemortalität.

Die Ernährung wird ebenfalls vielfach als ungenügend bezeichnet; so heisst es von Schwetzingen: „Wurst und Kartoffeln, oder Käse und Bier keine Mittagsmahlzeit für hart arbeitende Menschen. Für Eberbach berichtet Eberle: die Arbeiter nehmen grösstenteils ihre Mittagsmahlzeit ausserhalb der Wohnungen und infolgedessen zum grössten Teil kalt und geniessen dabei anstatt Milch oder nahrhafter Suppen minderwertiges Bier in nicht geringen Quantitäten.

Als Ursache dieser Zustände wird fast allgemein weniger die krasseste Armut und Not, die erst bei Arbeitsunfähigkeit der Familienernährer eintritt, sondern das Leben von der Hand in den Mund, ferner die Unkenntnis der Frauen in der Zubereitung geeigneter Nahrungsmittel beschuldigt. Schneider (Staufen) charakterisiert treffend diese "Unkenntnis in der Unterscheidung von Nahrungs- und Genussmitteln," so dass Bier und Kaffee als Nahrungsmittel genossen werden, letzterer sogar mittags als Hauptmahlzeit. Dass die jungen und alten Frauen von Hauswirtschaft nicht viel verstehen, ist sicher," führt der Wieslocher Berichterstatter Schleid aus, „wann sollten sie aber auch dazu kommen, da Nutter und Tochter zusammen in die Fabrik gehen! Nicht die Armut macht den Menschen krank, sondern die Geldgier." Das letztere dürfte wohl etwas paradox ausgesprochen sein; die Arbeit der Frauen in ausgedehntem Masse hängt vielfach mit der Niedrigkeit des Lohnes für männliche Arbeiter zusammen, 
so dass der Lohn des Familienvaters eben für das Bedürfnis der Familie vielfach nicht ausreicht. Andererseits drückt auch wieder die ausgedehnte Verwendung weiblicher Arbeiter den Lohn der männlichen herab. So zeigt Wörrishofer in seiner obenerwähnten Schrift, dass das Budget mancher Familien auch ohne grosse Bedürfnisse und Ansprüche mit einem Defizit abschliessen muss, wenn aus irgend einem Grunde sich nicht alle Familienmitglieder an dem Erwerbe beteiligen können.

Nach diesen, allerdings einer subjektiven Färbung nicht entbehrenden Angaben lässt sich die Möglichkeit eines direkteren Zusammenhangs zwischen der Armut und der in ihrem Gefolge erscheinenden Schädlichleitstrias, Unterernährung, enge Wohnung und Unreinlichkeit, nicht von der Hand weisen. Jedenfalls tritt aber der Einfluss in den auf statistischem Wege gewonnenen Zahlen für Baden nicht hervor. Für Berlin, Hamburg, München und Frankfurt am Main ist es Raths gelungen, ein verschiedenes Verhältnis der Tuberkuloseverbreitung in den von ärmeren Volksklassen bewohnten Stadtteilen gegenüber den von Wohlhabenderen bewohnten zu Ungunsten der ärmeren nachzuweisen. Hier scheint diese Tatsache mehr eine Folge der Berufsschädigung und vermehrten Infektionsgefahr der Arbeiterbevölkerung gegenüber den mehr geschonten Wohlhabenden zu sein, und es bleibt fraglich, ob für ländliche Bezirke, wo es auch für die ärmere Bevölkerung exquisit gesunde Berufsarten gibt, sich ähnliche Verhältnisse finden.

Ebenfalls zu nicht eindentigen Resultaten führte ein Versuch, die Ernährungsweise der Bevölkerung direkt zu kontrollieren. Als Massstab sollte dienen die Stiickzahl des während eines Jahres zum Konsum gekommenen Rindviehs pro Seelenzahl der Bevölkerung. Die von drei während des Zeitraumes 1891-1900 herausgegriffenen Jahren aus den Fleischbeschaulisten gewonnenen Durchschnittszahlen schwanken zwischen 13,9 Stück auf 100 Einwohner in Mannheim und 3,35 in Eppingen. Der Durchschnitt wird schätzungsweise bei 8-9 liegen. Diese Zahlen zeigen eine mit der Vermögenslage in entferntem Parallelismus stehende, unregelmässige Schwankung, so dass sich auch aus ihnen keine sicheren Schlüsse gestalten. Auch eine Korrektion derselben durch Berücksichtigung des verschiedenen Durchschnittsgewichtes des Rindviehs in verschiedener Gegend, das für den Gesamtrindviehbestand von $283 \mathrm{~kg}$ pro Stück im Kreise Lörrach bis zu $368 \mathrm{~kg}$ im Kreise Konstanz schwankt, führte zu keinem befriedigenden Resultate.

Ähnlichen Schwierigkeiten der Deutung begegneten den Zahlen, welche als Massstab für den Alkoholkonsum der Bevölkerung dienen 
sollten. Präcise Zahlen, welche direkt den Konsum der Bevölkerung wiedergeben, sind für den Weinkonsum zu erhalten aus der Weinsteuer, speziell dem Weinaccis, der von allem zum Konsum kommenden Wein erhoben wird. In Literzabl pro Kopf der Bevölkerung berechnet, ebenfalls aus drei willkürlich herausgegriffenen Jahren, ergaben sich Durchschnittszahlen, die von 12,2 1 in Eppingen bis zu 43,2 I in Karlsruhe und 54,5 I in Baden schwanken. Im allgemeinen entsprechen diese Zahlen den Vermögenszahlen; auch da, wo sich ein Gegensatz bemerkbar macht, wie z. B. in St. Blasien und Schönau, lässt er sich auf einfache Weise durch den beträchtlichen Konsum erklären, der seitens zugereister Gäste in den Luftkurorten während der Sommermonate geschieht. Auf gleiche Weise findet der enorme Konsum in Baden seine Deutung, wo allerdings auch ein grosser Teil des Verbrauchs auf die Bevölkerung fällt.

Unzuverlässigere Zahlen lassen sich für den Bierkonsum erhalten, da hier nur für die Steuerämter die Produktions-, Ausfuhr- und Einfuhrzahlen bekannt sind. Der ziemlich ansgedehnte Handel zwischen den verschiedenen Amtsbezirken entzieht sich der Beobachtung. Eine nähere Besprechung der gefundenen Zahlen würde zu keinem eindeutigen Ergebnis führen, doch sei ihnen ein Platz in Rubrik 19 der Tabelle I eingeräumt.

Auch aus der Zahl der Wirtschaften pro 100 Lebenden in den verschiedenen Amtsbezirken wurde versucht, eine Massgabe zu erhalten für den Alkoholkonsum, so dass sich aus Wein- und Bierkonsum und Wirtshauszahl ein zahlengemässes Bild von dem Wirtshausleben der Bevölkerung gewinnen liesse. Auch diese Zahlen lassen keinen Zusammenhang mit denjenigen für die Tuberkulosemortalität erkennen. Zur Entscheidung aber, ob aus dem Fehlen eines Zusammenhangs zwischen den als Massstab für den Alkoholkonsum und der Eirnährungsweise aufgestellten Zahlen und den Zahlen der Tuberkulosemortalität wirklich das Fehlen einer Beeinflussung zu schliessen ist, halte ich mich nicht für berechtigt. $\mathrm{Zu}$ diesem Zwecke müssten erst in kleinerem Kreise analytische Arbeiten vorausgehen, welche das Zutreffen der durch die erwähnten Zahlen im grossen dargestellten Verbältnisse im Detail bestätigten. Immerhin bieten sie auch in ihrer jetzigen noch umschleierten Form manche, das Interesse anregende Gesichtspunkte.

Zur besonderen Vorsicht in einer negierenden Schlussfolgerung wegen des Einflusses des Alkoholkonsums forderte die Wichtigkeit auf, mit der diese Frage in dem Berichte der Bezirksärzte behandelt wird. Hier beklagt der Bruchsaler Berichterstatter die üble Gewohnheit, in manchen Familien anstatt regelmässiger warmer Mahlzeiten 
vielfach das Geld zur Beschaffung von Bier und kalter Küche und häufig auch von Branntwein zu verwenden. In Ettlingen wird von Fröhlich die Unsitte gefunden, sogar kleinen Kindern Bier zu geben; das Gleiche gilt von Staufen, wo sogar bisweilen Säuglinge „als stark machend" Wein erhalten. Fast überall die Klage, dass der Alkoholkonsum in keinem Verhältnis steht zur Aufnahme von Nahrung. In Eberbach herrscht die Unsitte bei den Steinbrechern und Steinhauern, Bier fassweise, auf gemeinsame Kosten angeschafft, auf die Arbeitsplätze mitzunehmen, wodurch auch an und für sich mässige Leute, um auf die Kosten zu kommen, zu vermehrtem Trinken gebracht werden.

Es sind ja nun diese Berichte deshalb mit Zurückhaltung in ihrer Beurteilung zu behandeln, weil die entsprechenden und zum Vergleich heranzuziehenden aus den gesünderen Amtsbezirken nicht daneben gestellt sind, sondern nur von den in der Schwindsuchtsmortalität über Landesdurchschnitt stehenden der Bericht eingefordert wurde. Es wäre ja nun möglich, dass - dem Ergebnis der aufgestellten Statistik entsprechend - auch in diesen Gebieten dieselben Klagen über allzureichlichen Alkoholkonsum ertönten.

Eine Lösung dieses scheinbaren Widerspruchs wäre vielleicht ausserdem durch die Erwägung möglich, dass viele der erwähnten Missstände nicht als solche die Tuberkulose herbeiführen, sondern nur in ihren Folgezuständen. Diese wieder können gemeinsam für die verschiedensten Faktoren sein. Es ergibt sich schliesslich ein solches Gewirre sich gegenseitig beeinflussender Verhältnisse, dass eine Lösung und Schlichtung über die Kompetenz der statistisch abzählenden Änalyse geht.

\section{Einfluss der Berufsarten.}

Wir kommen nun zu dem wichtigsten Teil, zu der Beantwortung Allgemeine der Frage nach dem Einfluss der Beschäftigungsart auf die Tuber- Gunkste und kulosemortalität. Es fehlen, wie schon früher erwähnt, Statistiken über die Berufsart der Gestorbenen. Wir können mit dem uns zu Gebote stehenden Material nur so verfahren, dass wir die berufliche Zusammensetzung der lebenden Bevölkerung in Beziehung setzen zu der Mortalitätsziffer an Phthise. Die von uns aufgestellten Mortalitätsziffern sind also die Summe der Mortalitätsziffern der einzelnen Berufe und der beruflosen Angehörigen. Wie weit sie durch die über den Durchschnitt steigende Mortalitätsziffer eines Berufes beeinflusst werden können, ergibt sich aus der Mächtigkeit dieses Berufes in der Bevölkerung und aus der Zusammensetzung der dem Beruf an- 
gehörigen Bevölkerungsschicht aus wirklich Erwerbstätigen und deren Angehörigen. Bis sich an der Gesamtmortalität der Einfluss eines Berufszweiges mit erhöhter Mortalitätsziffer an Phthise bemerkbar macht, muss derselbe einen gewissen Prozentsatz im Gemenge der Berufe erlangt haben. Dann aber wird der Ausschlag in der Gesamtsterblichkeit nicht nur durch das Mehr an Tuberkulosetodesfällen bedingt sein, die dem Berufe selbst zur Last fallen, sondern auch durch die Opfer der Ansteckung durch Kranke dieses Berufes unter der übrigen Bevölkerung. Es liegt also in den auf diese Weise gefundenen Resultaten die Lösung der Frage nach der Gefahr bestimmter Berufszweige für die Gesamtbevölkerung. Diese Frage kommt besonders für das staatliche Interesse in Betracht. Denn für den Staat ist es von ganz verschiedener Wichtigkeit, ob ein Berufszweig zwar das Leben der damit Beschäftigten erheblich verkürzt, eine weitere Krankheitsverbreitung aber nur in beschränktem Masse stattfindet, oder ob ausser der Schädigung der Berufsthätigen noch eine Verbreitung in die Familie und weitere Kreise begünstigt wird. Bei letzterem spielen die mehr auf sozial-ökonomischem Gebiet liegenden Momente der Frauenarbeit, der Beschäftigung jugendlicher Arbeiter, der Mitarbeit der ganzen Familie eine bedeutende Rolle. Zum zahlenmässigen Ausdruck kommen diese Verhältnisse in den Prozentziffern für Erwerbsthätige, - getrennt in männliche und weibliche, -- und Zahlen der Angehörigen und Dienenden. Bei den weiblichen Erwerbsthätigen wäre wieder zu unterscheiden der Familienstand, ob ledig oder verheiratet. Es sind dies Ziffern, welche bei gegebener Infektionsgelegenheit durch den Beruf, Rückschlüsse zulassen auf die Gefahr des Einschleppens in Familie, oder bei einer direkten Schädigung durch den Beruf, den Einfluss desselben auf die Familie abschätzen lassen.

Dass die Folgen eines grösseren oder kleineren Verdienstes bei verschiedenen Berufsarten in ihren Rückschlägen auf Wohnungsverhältnisse und Ernährung und dergleichen sich äussern, bedarf keiner weiteren Klarlegung. Zu dieser Verschiedenheit des Verdienstes der Berufsarten kommt noch eine Verschiedenheit nach der Gegend. Diese Verschiedenheiten aber zahlenmässig zu fixieren, erwies sich bei den vielfach ungenauen, oft nur für juristische Streitfragen ausschlaggebenden, den wirklichen Löhnen nicht entsprechenden Angaben als ein fruchtloses Unternehmen.

Verfolgen wir nun in einer dem Schema der statistischen Ämter entsprechenden Reihenfolge die einzelnen Berufsgruppen und Arten in ihrer zahlenmässigen Zusammensetzung nach der Berufszählung 
vom 14. Juni 1895 und wenden uns zunächst zu der Betrachtung der Hauptgruppen :

A. Landwirtschaft und Tierzucht, Gärtnerei, Forstwirtschaft, Jagd und Fischerei.

B. Gewerbe und Industrie mit Einschluss des Bergbaues, der Hütten und Salinen, sowie des Bauwesens.

C. Handel, Versicherungswesen und Verkehr.

D. Taglohnarbeit wechselnder und gemischter Art, sowie persönliche Dienstleistung.

E. Militär, Hof-, bürgerlicher und kirchlicher Beruf, auch sogenannte freie Berufsarten.

F. Beruflose, d. h. Personen ohne Berufsausübung, Rentner, Pensionäre, von Unterstützung lebende, Zöglinge, Studierende, Insassen von Irren-, Straf- und Besserungsanstalten und Personen ohne Berufsangabe.

Zur Orientierung über die Schwankungen in der Zusammensetzung der Bevölkerung aus diesen Berufsgruppen in Prozentzahlen, welche die Angehörigen der einzelnen Gruppen mit umfassen, diene folgende Übersicht.

\begin{tabular}{|c|c|c|c|c|c|c|}
\hline $\begin{array}{c}\text { Bezeichnungen der } \\
\text { Berufsgruppen }\end{array}$ & A & B & C & D & E & F \\
\hline $\begin{array}{c}\text { Minimum } \\
\text { im Amtsbezirk }\end{array}$ & 7,37 & 13,0 & 4,5 & 0,178 & 2,43 & 2,64 \\
\hline $\begin{array}{c}\text { Landesdurchschnitt } \\
\text { Maximum }\end{array}$ & 42,3 & 34,8 & 9,95 & 0,798 & 5,49 & 6,55 \\
\hline im Amtsbezirk & 71,8 & 61,2 & 26,0 & 2,25 & 13,0 & 14,82 \\
\hline
\end{tabular}

Einer eingehenderen Besprechung sollen die Gruppen A als Landwirtschaft charakterisiert, $B=$ Industrie, $C=$ Handel unterzogen werden. Die übrigen drei Gruppen sind von geringerer Wichtigkeit, einmal wegen ihrer geringen zahlenmässigen Grösse, so erhebt sich Gruppe D, die der wechselnden Taglohnarbeit etc. nicht über eine Prozentbeteiligung, bei welcher man eine Beeinflussung mit Aussicht auf irgend einen Erfolg nachzuweisen versuchen könnte, die Grösse der Gruppe E ist hauptsächlich durch die Grösse der Garnisonen bedingt, und die Zusammensetzung der letzten Gruppe ist zu verschiedenartig aus den mannigfachsten Kategorien, als dass man sie in den verschiedenen Bezirken einheitlich bewirten könnte. 
Betrachten wir zunächst die Zusammensetzung der einzelnen Berufsgruppen aus Frwerbstätigen - Männern und Frauen - Dienstboten und Angehörigen an Hand der nebenstehenden Tabelle.

\begin{tabular}{|c|c|c|c|c|}
\hline \multicolumn{2}{|c|}{$\begin{array}{l}\text { Bezeichnung der } \\
\text { Berufsgruppe }\end{array}$} & $\frac{\text { A }}{\text { Landwirtsch. }}$ & $\begin{array}{c}\text { B } \\
\text { Industrie }\end{array}$ & $\begin{array}{c}\mathrm{C} \\
\text { Handel }\end{array}$ \\
\hline \multirow{3}{*}{ 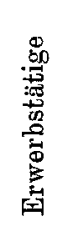 } & Gesamt & 51,1 & 48,2 & 44,1 \\
\hline & Männer & 31,6 & 36,2 & 30,2 \\
\hline & Frauen & 19,5 & 12,0 & 13,9 \\
\hline \multicolumn{2}{|c|}{ Dienstboten } & 1,44 & 2,12 & 5,77 \\
\hline \multicolumn{2}{|c|}{ Angehörige } & 47,46 & 49,68 & 56,03 \\
\hline
\end{tabular}

Die Verschiedenheit der Zusammensetzung in den einzelnen Gruppen ist sofort evident. Bei Folgerungen auf den Einfluss derselben hinsichtlich der Tuberkulosehäufigkeit ist $\mathrm{zu}$ unterscheiden, ob es sich um direkt schädliche Berufe, oder solche mit vermehrter Infektionsgefahr handelt, schliesslich kann sich auch beides vereinigen. Dann wird eine grössere Beteiligung der Frauen einen entschiedenen Nachteil bedeuten, da zu der Gefahr der Infektion in der Familie und im Haus noch die Berufsschädlichkeiten mit ihren Infektionsquellen und Gefahren hinzutreten. Andererseits bei gesunden Berufen, die keine besonderen Infektionsgefahren herbeiführen, ist ein Zuzug der Frauen nicht schädlich anzusehen, eher sogar vorteilhaft, da die Ansteckungsgefahr in der Familie herabgesetzt wird, insbesondere zu Hause liegende Kranke auf kleinere Kreise ansteckend wirken können. Als Beispiel für letztere Kategorie mag die Landwirtschaft gelten, die eine Beteiligung von 19,5 Prozent Frauen unter den Erwerbstätigen aufweist.

Fine Einschränkung dieser Behauptungen ist für diejenigen Berufsarten geboten, bei denen die verheirateten Frauen in grosser Zahl beteiligt sind, abgesehen von der Landwirtschaft. Es wird durch die Berufsarbeit die Mutter den Kindern entzogen, diese wesentlich der Aufsicht der Alten und Kranken überlassen, die Reinlichkeit des Haushaltes, die Zubereitung der Speisen leidet not, und es werden alle jene Zustände begünstigt, welche bei Erkranktsein eines Familien- 
gliedes eine weitergreifende Familieninfektion, namentlich der heranwachsenden Generation begünstigen. Diese Missstände erfahren selbstverständlich die grössten Schwankungen bei den verschiedenen Berufszweigen der Industrie; es erweist sich daher nötig diese einzeln abzuhandeln. Vorläufig sei nur so viel als sicher festgestellt, dass die Gesamtheit der Industriebeschäftigten als mehr gefährdet angesehen werden muss als die landwirtschaftlichen Berufszweige. Für die Frauenarbeit hat die Landwirtschaft auch mit aus dem Grunde eine Sonderstellung, weil im landwirtschaftlichen Betrieb die Frau viele sie in das Haus zurückführende Beschäftigungen hat, wobei ihr Aufenthalt im Hause in natürlicher Weise der Erfüllung ihrer Hausfrauen- und Mutterpflichten za Gute kommt.

Diese theoretische Behauptung einer geringeren Infektionshäufig- Landwirtkeit für den landwirtschaftlichen Beruf im ganzen stimmt überein Industrie. mit der von Köhler (7) angeführten Statistik des Reichsgesundheitsamtes, wonach die Zahl der Invalidenrenten, welche wegen Tuberkulose bewilligt werden, in der Industrie durchweg höher sind als in der Landwirtschaft, und mehr als das dreifache erreichen können. Wenn demgegenüber R e ib m a y $(17)$ anführt, ein in seinem Sinne disponierter, d. h. mit latenter Tuberkulose kämpfender Mensch gehe bei der anstrengenden Arbeit, wie sie die Landwirtschaft erfordert, schneller zu Grunde, als bei leichter Industriebeschäftigung auch unter an sich ungünstigeren hygienischen Bedingungen und könne sich so eine gewisse Immunität erwerben, so kann er gegen die Beweiskraft dieser Zahlen nicht ankämpfen, er müsste bei Aufrechterhaltung seiner Theorie zu dem Schlusse gedrängt werden, dass in der landwirtschạftlichen Bevölkerung der Zahl dieser "latent Tuberhuloser" geringer ist. Überdies dürfen wir nie vergessen, dass die Reibmayrschen Sätze Folgerungen einer Hypothese sind, die eben Hypothese bleibt, so geistreich sie auch erdacht und durchgeführt ist.

Kommen wir nun zu der Antwort, welche uns die statistische Zusammenstellung der Berufsverbreitung mit der Tuberkulosehäufigkeit für Baden auf diese Frage erteilt. Auf Tabelle I sind die Ziffern der Zusammensetzung der Bevölkerung nach den verschiedenen Berufsgruppen in Prozentziffern der Gesamtbevölkerung für die einzelnen Amtsbezirke angegeben, auf Tafel II findet sich die entsprechende graphische Darstellung. Die Reihenfolge ist auf beiden Wiedergaben übereinstimmend durch das Ansteigen der Tuberkulosemortalität gegeben. Es zeigt sich nun in grossen Zügen mit Zunahme der Industrieberölkerung und Abnahme der Landwirtschaft eine Zunahme der Mortalitätsziffern an Tuberkulose. Desgleichen findet sich zahlreiche 
handeltreibende Bevölkerung in den Amtsbezirken mit hoher Tuberkulosemortalität.

Es könnte nun der Einwurf erhoben werden, nicht die $\mathrm{Zu}$ sammensetzung der Bevölkerung ist in primären Zusammenhang zu bringen mit der Tuberkulosemortalität, sondern diese Zusammensetzung selbst ist Folge oder Begleiterscheinung von Umständen, welche diesen Einfluss auf die Tuberkulosesterblichkeit besitzen, z. B. der Höhenlage. Denn aus der gleichen Tafel II können wir ein Absinken der Industriebevölkerung und Zunahme der landwirtschaftlichen Bevölkerung mit Zunahme der Durchschnittshöhe der Amtsbezirke über dem Meeresspiegel ersehen. Wenn dem so wäre, müsste für Gruppen von Amtsbezirken von ähnlicher Höhe die $\mathrm{Zu}-$ sammensetzung der Bevölkerung keinen Einfluss erkennen lassen auf die Mortalitätszahlen an Schwindsucht.

Betrachten wir mit Rücksicht auf diese Verhältnisse die detaillierte Darstellung der Amtsbezirke in vier Höhengruppen auf Tabelle II und Tafel IV. Es zeigt sich in jeder einzelnen Höhengruppe in analoger Weise wie bei der Gesamtaufstellung für das ganze Land eine Zunahme der Industriebevölkerung und Abnahme der landwirtschaftlichen Bevölkerung.

Es bieten aber auch die vier Höhengruppen als Ganzes nebeneinander betrachtet manches Bemerkenswerte. Eine kurze Übersicht sei hier wiedergegeben.

\begin{tabular}{|c|c|c|c|c||}
\hline $\begin{array}{c}\text { Bezeichnung } \\
\text { der } \\
\text { Höhengruppe }\end{array}$ & $\begin{array}{c}\text { I } \\
100-200 \\
\mathrm{~m}\end{array}$ & $\begin{array}{c}\text { II } \\
200-300 \\
\mathrm{~m}\end{array}$ & $\begin{array}{c}\text { III } \\
300-500 \\
\mathrm{~m}\end{array}$ & $\begin{array}{c}\text { IV } \\
\text { über 500 } \\
\mathrm{m}\end{array}$ \\
\hline $\begin{array}{c}\text { Landwirt- } \\
\text { schaft }\end{array}$ & 35,2 & 60,3 & 41,6 & 52,8 \\
\hline Industrie & 40,9 & 25,3 & 38,1 & 31,6 \\
\hline $\begin{array}{c}\text { Tuberkulose- } \\
\text { mortalität }\end{array}$ & 3,18 & 2,56 & 2,58 & 2,06 \\
\hline
\end{tabular}
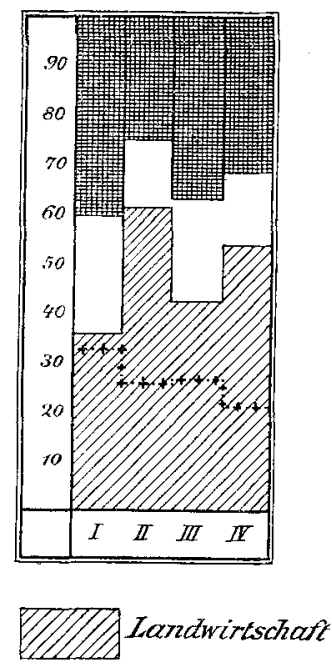

Es zeigt sich im allgemeinen in diesen Höhengruppen mit Zanahme der Höhe eine Zunahme der Landwirtschaft und Abnahme der Industrie mit Absinken der Tuberkulosemortalität. Eine Ausnahme macht nur die zweite Höhengruppe. Diese sollte ihrer Höhenlage nach eine höhere Tuberkulosemortalität erwarten lassen als die 
dritte Gruppe. Es zeigt sich aber das Gegenteil, die Höhengruppe II hat eine, wenn auch nur wenig höhere Tuberkulosemortalität als Gruppe III. Dieses mit Rücksicht auf die Höhenlage atypische Verhalten muss durch andere Faktoren bedingt sein, und als ein solcher drängt sich der Kritik die geringe Industriebeteiligung und das Vorwiegen des Ackerbaues in Gruppe II geradezu auf. Ein Unterschied von nahezu $20 \%$ in der Landwirtschaft mehr, und über $12 \%$ weniger in der Industrie muss sich bemerkbar machen und überwiegt den in diesem Falle umgekehrt wirkenden Einfluss der Höhenlage.

Wenn wir nun im Vorhergehenden einen entschiedenen Einfluss Berufsarten der Zusammensetzung der Bevölkerung aus Ackerbau- und Industrie- Industrie treibenden auf die Tuberkulosemortalität feststellen konnten, so reizt es zur Aufrollung der weitergehenden Frage, ob sich nicht auch auf gleichem Wege ein Einfluss bestimmter Zweige der Industrie nachweisen liesse. Für diese Untersuchung ist die für die statistischen Feststellungen gewählte Einteilung im Amtsbezirke entschieden ungünstig. Wenn auch die Amtsbezirke einigermassen einheitliche Komplexe darstellen in ihrer Bevölkerungszusammensetzung aus Ackerbau und Industrietreibenden, so können doch in den einzelnen Orten die grössten Verschiedenheiten einzelner Berufszweige bestehen, vom vollständigen Fehlen bis zum fast ausschliesslichen Vorhandensein. Für den gesamten Amtsbezirk gleichen sich aber diese Unterschiede bereits wieder aus. Es können daher diese Untersuchungen nur für einige wenige Berufsgruppen, die diesen Nachteilen weniger, ausgesetzt sind, ausgeführt werden; für die Mehrzahl muss auf eine ausführliche Besprechung verzichtet werden.

Erwägungen in Betreff des Einflusses der Zusammensetzung der Berufsgruppen aus Erwerbsthätigen, Männern und Frauen und nicht im Beruf beschäftigten Angehörigen sind auch hier anzustellen und diesen Faktoren häufig eine grosse Wichtigkeit beizulegen. Zur Veransehaulichung dieser Unterschiede diene untenstehende l'abelle, auf der sich in Spalte vier bis sechs die Zahlen finden, welche den Anteil der betreffenden Berufsgruppe an der Bevölkerung angeben, in Spalte sieben bis zehn die Zahlen, welche die Zusammensetzung der Berufsgruppe oder -art charaktėrisieren. Bei der ersten Gruppe von Zahlen finden sich die Angaben über Landesdurchschnitt und Minimum und Maximum im Amtsbezirk, in der zweiten Zahlengruppe die Detaillierung der Berufsgruppen in Erwerbstätige, - Männer und Frauen, - häusliche Dienstboten und Angehörige. Die Zahlen sind prozentuarische Verhältniszahlen zu der Gesamtzahl des betreffenden Berufes in ganz Baden. Zum Vergleich ist die Tabelle auch noch einmal für die Hauptberufsgruppen, Aekerbau, Industrie, Handel und Verkebr u. s. w. durchgeführt. 
Berufsgruppen, Abteilungen und Arten in ihrer Beteiligung an der Zusammensetzung der Bevölkerung Badens und ihrer Zusammensetzung aus Erwerbsthätigen etc.

\begin{tabular}{|c|c|c|c|c|c|c|c|c|c|}
\hline \multirow{3}{*}{ 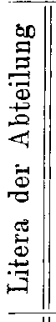 } & \multirow{3}{*}{ 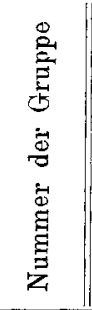 } & \multirow{3}{*}{$\begin{array}{l}\text { Berufsabteilungen, } \\
\text { Gruppen und Arten }\end{array}$} & \multicolumn{3}{|c|}{$\begin{array}{c}\text { Zahlen der dem betreffenden } \\
\text { Beruf Angehörigen in \% der } \\
\text { Bevölkerung }\end{array}$} & \multicolumn{4}{|c|}{$\begin{array}{c}\text { Zusammensetzung der } \\
\text { Berufsgruppen in Prozent- } \\
\text { zahlen ihrer Gesamtzahlen } \\
\text { aus: }\end{array}$} \\
\hline & & & \multirow{2}{*}{$\begin{array}{l}\text { Minimum } \\
\quad \text { im } \\
\text { Amtsbezirk }\end{array}$} & \multirow{2}{*}{ 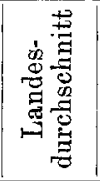 } & \multirow{2}{*}{$\begin{array}{c}\text { Maximum } \\
\text { im } \\
\text { Amtsbezirk }\end{array}$} & \multicolumn{2}{|c|}{$\begin{array}{c}\text { Erwerbs- } \\
\text { thätige }\end{array}$} & \multirow{2}{*}{ 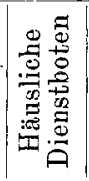 } & \multirow{2}{*}{ 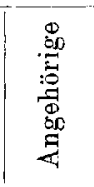 } \\
\hline & & & & & & 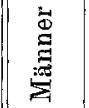 & 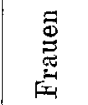 & & \\
\hline 1 & 2 & 3 & 4 & 5 & 6 & 7 & 8 & 9 & 10 \\
\hline A & I u. II & $\begin{array}{l}\text { Landwirtsch., Gärt- } \\
\text { nerei u. Tierzucht, } \\
\text { Forstwirtsch., Jagd } \\
\text { und Fischerei.. }\end{array}$ & $\begin{array}{c}\text { Mannheim } \\
7,37\end{array}$ & 42,4 & $\begin{array}{l}\text { Breisach } \\
71,8\end{array}$ & 31,7 & 19.3 & 1.44 & 47,56 \\
\hline \multirow[t]{17}{*}{ B } & III & $\begin{array}{l}\text { Bergbau, Hütten, Sa- } \\
\text { linen, Torfgruben . }\end{array}$ & 0 & 0,066 & $\begin{array}{l}\text { Sinsheim } \\
1,315\end{array}$ & 31,8 & 1,2 & 1,68 & 66,43 \\
\hline & IV & $\begin{array}{l}\text { Industrie der Steine } \\
\text { und Erden . . . }\end{array}$ & $\begin{array}{l}\text { Schönau } \\
0,2725\end{array}$ & 2,209 & $\begin{array}{l}\text { Eppingen } \\
8,86\end{array}$ & $\mid 38,49$ & 2,41 & 1,13 & 57,97 \\
\hline & & davon Steinmetzen & $\begin{array}{l}\text { Wiesloch } \\
0,0888\end{array}$ & 0,527 & $\begin{array}{c}\text { Eppingen } \\
6,67\end{array}$ & 41,3 & 0,43 & 0,575 & 57,695 \\
\hline & $\mathrm{V}$ & Metallverarbeitung. & $\begin{array}{l}\text { Bonndorf } \\
1,025\end{array}$ & 4,45 & $\begin{array}{l}\text { Pforzheim } \\
36,0\end{array}$ & 39,77 & 5,83 & 1,67 & 52,73 \\
\hline & VI & $\begin{array}{l}\text { Masebinen, Werk- } \\
\text { zeuge,Instrumente, } \\
\text { Apparate. . . }\end{array}$ & $\begin{array}{c}\text { Breisach } \\
0,372\end{array}$ & 2,344 & $\begin{array}{l}\text { Triberg } \\
22,4\end{array}$ & 39,49 & 2,61 & 2,31 & 55,59 \\
\hline & VII & Chemische Industrie & $\begin{array}{r}\text { Staufen } \\
0,03283\end{array}$ & 0,631 & $\begin{array}{c}\text { Sehwetzingen } \\
4,505\end{array}$ & 30,9 & 13,4 & 5,16 & 50,54 \\
\hline & VIII & $\begin{array}{l}\text { Forstwirtschaftliche } \\
\text { Nebenprodukte, } \\
\text { Leuchtstoffe, Fette, } \\
\text { Öle, Firnisse. . }\end{array}$ & $\begin{array}{c}\text { Adelsheim } \\
0,00713\end{array}$ & 0,2715 & $\begin{array}{l}\text { Mannheim } \\
1,039\end{array}$ & 32,64 & 3,56 & 3,79 & 60,01 \\
\hline & IX & T'extilindustrie . & $\begin{array}{l}\text { Wiesloch } \\
0,0755\end{array}$ & 2,51 & $\begin{array}{l}\text { Säckingen } \\
23,2\end{array}$ & $\mid 28,0$ & 34,0 & 1,42 & 36,58 \\
\hline & $\mathrm{X}$ & Papierindustrie & $\begin{array}{c}\text { Eberbach } \\
0,0466\end{array}$ & 0,82 & $\begin{array}{l}\text { Lahr } \\
3,83\end{array}$ & 34,9 & 12,5 & 2,13 & 50,47 \\
\hline & $\mathrm{XI}$ & Lederindustrie & $\begin{array}{l}\text { Breisach } \\
0,1712\end{array}$ & 0,97 & $\begin{array}{l}\text { Weinheim } \\
\mathbf{1 1 , 9 3}\end{array}$ & || $38,06 \mid$ & 4,14 & 2,28 & 55,52 \\
\hline & XII & $\begin{array}{l}\text { Holz- und Schnitz- } \\
\text { stoffe } . \\
\end{array}$ & Tanberbischofsh. & 3,15 & $\begin{array}{c}\text { Schönau } \\
11,94\end{array}$ & 39,71 & 2,89 & 1,5 & 55,9 \\
\hline & & $\begin{array}{l}\text { davon Bürsten- } \\
\text { macher } .\end{array}$ & & 0,1938 & $\begin{array}{c}\text { Schönau } \\
9,57\end{array}$ & & 18,4 & 1,86 & 44,44 \\
\hline & XIII & $\begin{array}{l}\text { Nahrungs- und Ge- } \\
\text { nussmittel }\end{array}$ & $\begin{array}{c}\text { Emmendingen } \\
1,56\end{array}$ & 5,54 & $\begin{array}{l}\text { Wieslocb } \\
27,0\end{array}$ & 34,5 & 20,5 & 4,4 & 40,6 \\
\hline & & $\begin{array}{l}\text { davon Tabakfabri- } \\
\text { kation . } . . .\end{array}$ & 0 & 2,14 & $\begin{array}{l}\text { Wiesloch } \\
24,55\end{array}$ & 26,9 & 44,0 & 1,22 & 27,88 \\
\hline & XIV & $\begin{array}{l}\text { Bekleidung u. Reini- } \\
\text { gung } . \cdot \cdot \text {. }\end{array}$ & $\begin{array}{l}\text { Breisach } \\
2,47\end{array}$ & 4,96 & $\begin{array}{l}\text { Mannheim } \\
7,67\end{array}$ & 27,5 & 28,2 & 1,18 & 43,12 \\
\hline & $\mathrm{XV}$ & Baug & $\begin{array}{l}\text { Ettenheim } \\
\quad 3,06\end{array}$ & 6,19 & $\begin{array}{l}\text { Baden } \\
9,05\end{array}$ & 40,84 & 0,406 & 1,45 & 57,3 \\
\hline & XVI & $\begin{array}{l}\text { Polygraphisches Ge- } \\
\text { werbe . } .\end{array}$ & $\begin{array}{c}\text { Mosbach } \\
0,0302\end{array}$ & 0,45 & $\begin{array}{l}\text { Lahr } \\
2,68\end{array}$ & 44,17 & 5,43 & 3,36 & 47,04 \\
\hline & & & & & & II & & & \\
\hline
\end{tabular}




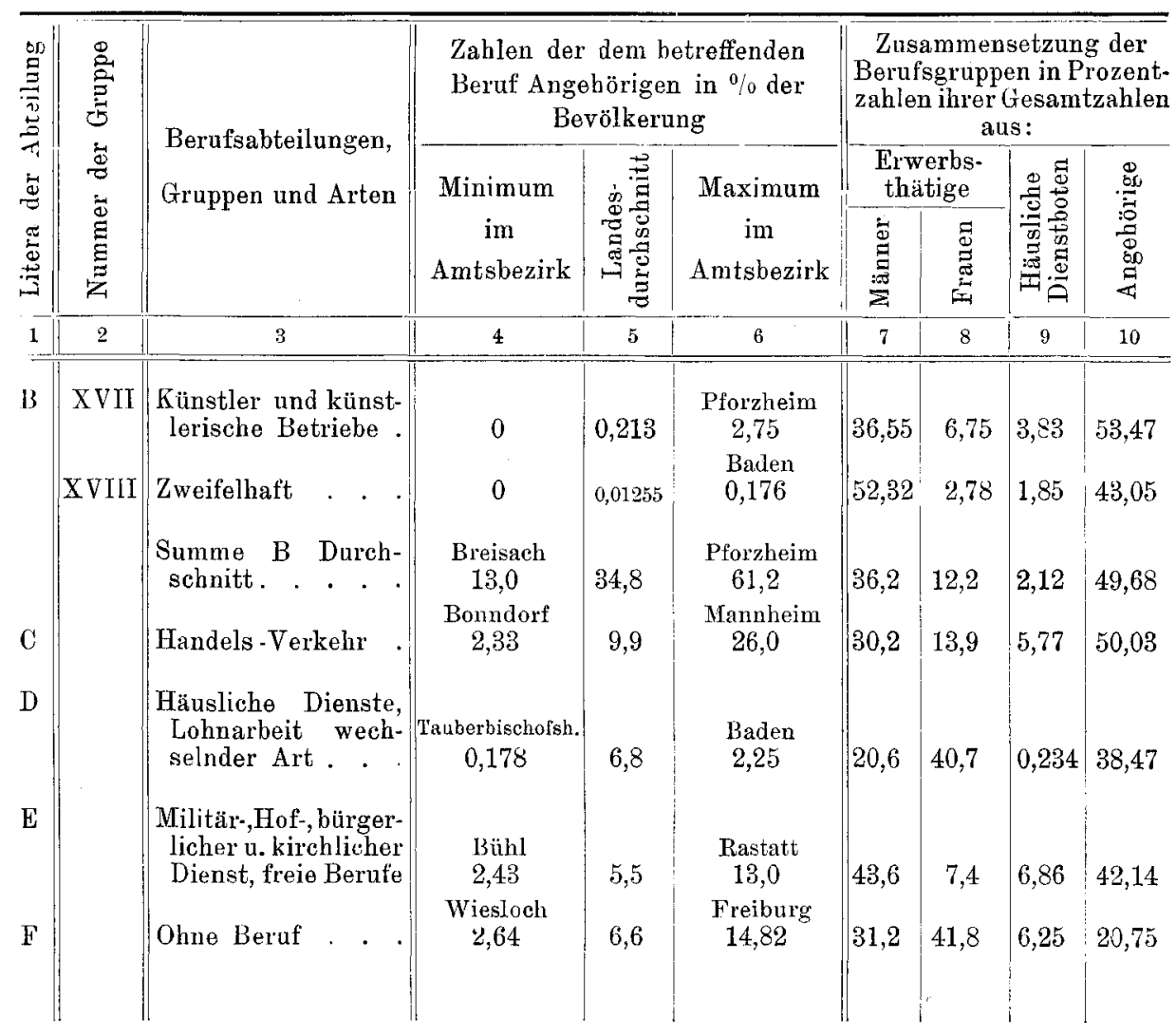

In beiden Abteilungen finden sich die erheblichsten Differenzen für die einzelnen Berufe sowohl, als in ihrer Beteiligung an der $\mathrm{Zu}-$ sammensetzung der Bevölkerung in den verschiedenen Amtsbezirken. Bis zu $36 \%$ der Berölkerung eines Amtsbezirkes gehört z. B. einem Beruf an, wobei derselbe allerdings vielgestalte Spezialberufe umfasst. Es ist dies die Metallverarbeitung in Pforzheim, die nächst höchste Zahl ist aber die vollkommen einheitliche Tabakfabrikation mit über $24 \%$ in Wiesloch. Der Anteil der nicht im Beruf beschäftigten Angehörigen an der Gesamtzahl schwankt von $27,88 \%$ ebenfalls in der Tabaksfabrikation bis zu $60,01 \%$ in der Industrie der forstwirtschaftlichen Nebenprodukte. Es zeigen sich bei der Tabaksindustrie diese beiden Faktoren, der eine, der hohe Prozentsatz in der Bevölkerung, welcher eine vermehrte Schwindsuchtsmortalität der Tabaksarbeiter zum stärkeren Ausdruck in der Gesamtmortalität bringt, und der zweite, die allgemeine Mitarbeit der Familien, welche die Infektionsgelegenheit auf den Arbeitsplätzen in 
den weitesten Kreisen verallgemeinert, besonders ausgeprägt. Dem entspricht auch vollkommen die Stellung der Amtsbezirke mit hoher Tabaksfabrikation unter den mit Phthise am stärksten durchseuchten. Ähnliche Ziffern in Betreff der Bevölkerungszusammensetzung kann nur noch die Textilindustrie bieten mit $23,4 \%$ der Bevölkerung in Säckingen und nur 36,58\% nicht Erwerbsthätigen. Bei der Textilindustrie ist aber eine Infektionsgefahr lange nicht so gross als bei der Tabaksindustrie infolge der beträchtlicheren räumlichen Trennung und der freieren Beweglichkeit der Arbeiter im Arbeitsraum. Einen hier vielleicht auch mitspielenden Faktor, den verschiedener Volks. rassen in den Tabaks- und Textilindustriegebieten, werden wir später noch diskutieren. Für eine grosse Anzahl der in der Tabelle erwähnten Gewerbe lässt sich natürlich ein ähnlicher Nachweis nicht erbringen, da ihre Beteiligung an der Bevölkerungszusammensetzung überhaupt, oder die Schwankungen in den einzelnen Amtsbezirken zu gering sind, oder schliesslich sie in hygienischem Sinne zu verschieden bewertbare Beschäftigungsweisen enthalten. Für einige wichtigere Berufe sind die Prozentsätze der Gesamtberufsklasse in der Bevölkerung, sowie in roten Zahlen der Erwerbstätigen allein für die einzelnen Amtsbezirke in Tabelle I Spalte 7-12 angegeben. Es zeigen sich nun diejenigen Amtsbezirke, in welchen einzelne Berufe besonders stark vertreten sind oder einige wenige zusammen einen grösseren Prozentsatz der Bevölkerung darstellen unter denjenigen, welche bedeutende Tuberkulosemortalität aufweisen. Ebenso ]ässt sich für die Amtsbezirke, welche hohe Mortalitätsziffern haben, ein starker Prozentsatz direkt schädlicher Berufe oder solcher, von denen wir einen ungünstigen Einfluss auf die Weiterverbreitung der Tuberkulose annehmen dürfen, nachweisen.

Im einzelnen zeigen die verschiedenen Berufsarten noch Beeinflussungen durch die mannigfachsten Besonderheiten. Es sei deshalb versucht, eine Besprechung der Gruppen noch im einzelnen zu geben, welche mit Bezug auf ihren Einfluss auf der Tuberkuloseverbreitung am meisten diskutiert worden sind.

In der Industrie der Steine und Erden ist für die Unterabteilung der Steinmetzen die schädliche Einwirkung des Steinstaubes auf die Lungen eine allgemein anerkannte Tatsache. So sterben nach Sommerfeld (22) 35 auf 1000 lebende Steinmetzen an Phthise, und das durchschnittliche Lebensalter schwankt zwischen 36 und 45 Jahren.

Die Art der Erwerbsthätigkeit bedingt eine nur geringe Beteiligung der Frauen, der sich für Baden mit nur 0,43\% darstellt. Auch von diesen ist keine Arbeit am Stein anzunehmen, sondern sie figurieren als Geschäftsinhaberinnen, Buchführerinnen eventuell auch als Zeich- 
nerinnen. Die Infektionsgelegenheit findet sich meist ausserhalb des Arbeitsplatzes, da sich dieser meist im Freien oder in nur einseitig geschlossenen Schuppen befindet, und weite Entfernungen die einzelnen Arbeiter trennen. Die Infektion ist daher vielfach Familieninfektion. Eine Verschiedenheit derselben für Mann und Frau besteht darin, dass die Frauen durch die Pflege der Kranken, und ihren längeren Aufenthalt im Hause den Infektionsquellen in erhöhtem Masse ausgesetzt sind als die Männer. Trotzdem ist eine häufigere Erkrankung der Männer zu beobachten; diese ist der Ausdruck der Schädigung durch den Steinstaub, der trotz anscheinend gegenüber den Frauen verminderter Infektionshäufigkeit eine Mehrerkrankung und grössere Mortalitätsziffern für die Männer herbeiführt. Für einen Ort von mehr als 1800 Einwohner, wo diese Verbältnisse durch eigene Untersuchungen ermittelt wurden, stellt sich ein Unterschied von 2,6\% zu Gunsten der Franen heraus.

Da dieser Beruf an das geographische Vorkommen brauchbarer Gesteinsarten gebunden ist, die sich oft nur auf kleine"Bezirke beschränlst finden, so treffen wir zerstreut Ortschaften, deren ganze Industriethätigkeit fast allein diesem Berufszweig gewidmet ist. Diese Orte sind häufig als "Schwindsuchsnester" landeskundig. In den einzelnen Amtsbezirken insgesamt erreicht dieser Beruf nur selten eine so hohe Prozentziffer, dass man Beziehungen zur Mortalitätsziffer der Gesamtbevölkerung an Tuberkulose aufstellen könnte. Es fällt aber sofort die starke Verbreitung der Steinhauerei in Eppingen auf, 2,45 Erwerbsthätige, mit Angehörigen 6,67\% der Bevölkerung, in Eppingen, einem Amtsbezirk, der günstige Gesundheitsverhältnisse darbietet. Der Verbreitung der Steinhauerei entsprechend, hätte man bedeutend höhere Mortalitätsziffern erwarten sollen. Nun ist aber in Eppingen die Steinhauerei der einzig bedeutendere Industriezweig, die Gesamtindustrie erreicht nur eine Beteiligung von $27 \%$ in der Bevölkerung, so dass ihr ungünstiger Einfluss durch den günstigen der stark verbreiteten Landwirtschaft weit überkompensiort wird.

Ausserdem 'ist die Verarbeitung der verschiedenen Gesteinsarten von äusserst verschiedenem Einfluss auf die Atmungsorgane. Granit und Kalkstein haben überhaupt fast keinen nennenswerten Einfluss, und auch der Keupersandstein der Eppinger Gegend ist in keiner Weise in seiner Schädigung der Atemorgane dem Buntsandstein der Wertheimer und Eberbacher Steinbrïche gleichzusetzen. Dort zeigt sich in den Steinhauerorten eine erschreckende Tuberkulosehäufigkeit, z. B. ergab sich für das im Bezirk Wertheim gelegene, einer genaueren Analyse unterzogene Freudenberg im Zeitraum 1870-1900 
eine Durchschnittsmortalität an Tuberkulose (Schwindsucht und anderweitigen tuberkulösen Krankheiten) von 9,3 auf 1000 Lebende männlichen Geschlechts, und 6,7 weiblichen. Ähnliche, zum Teil noch höhere Zahlen finden sich an anderen Orten. Für mehr als die Hälfte der Erwachsenen ist die Tuberkulose die Todesursache. Derartig schwer durchsenchte, vielfach als Enklave in gesunder Gegend liegende Ortschaften, eignen sich in rorzüglicher Weise für den Anfang einer auf Ortsanalysen aufgebauten statistischen Forschung. Der erste tastende Versuch einer solchen, an dem schon erwähnten Freudenberg ausgeführt, soll gewissermassen den zweiten Teil dieser nur allgemeineren und vorbereitenden Arbeit bilden.

Wenden wir uns nun zur Betrachtung der Tabaksindustrie. Ich schliesse mich zunächst den allgemeinen Ausführungen an, die Wörrish of er (30) der Arbeit iiber die soziale Lage der Tabaksarbeiter vorausschickt. Die Tabaksindustrie hat ihre Hauptverbreitung in den am wenigsten fruchtbaren Teil der Rheinebene und in wenigen Orten des angrenzenden Hügellandes. Vorzugsweise handelt es sich dabei um zwei geschlossene Komplexe; der grössere derselben liegt in der badischen Pfalz - den Amtsbezirken Schwetzingen, Wiesloch, Bruchsal und den angrenzenden Teilen der Amtsbezirke Heidelberg, Mannheim, Weinheim und Sinsheim entsprechend. Der andere weniger dicht besetzte Bezirk umfasst die Rheinebene zwischen Offenburg und Freiburg, vorzugsweise die Amtsbezirke Emmendingen, Ettenheim und die Stadt Lahr. In diesen Bezirken gehören etwa $5-25 \%$ der Bevölkerung den Cigarrenarbeitern an.

Bei der grossen Zahl der Erwerbstätigen in den Cigarrenarbeiterfamilien - ca. $70 \%$ - bei der starken Beteiligung der Frauen, auch der verheirateten, - $44 \%$ der Gesamtheit - ist es verständlich, dass bei Mängeln in der Verhütung von Infektionsgefahr auf den Arbeitsplätzen eine schnelle Verbreitung der Tuberkulose stattfinden kann. Ein Vergleich der Karten über Tuberkuloseverbreitung und Verbreitung der Tabaksindustrie zeigt nun weitgehende Übereinstimmung.

Eine besonders augenfällige Schädlichkeit ist durch die Eigenart des Materials soweit ersichtlich nicht gegeben, sondern durch die Art der Erledigung der Arbeit, der unglücklichen Kombination der arbeitenden Personen und der grossen Verbreitung dieses Berufszweiges dort, wo er überhaupt ernstlich betrieben wird. Es sind also keine prinzipiell eigenartigen Faktoren, wie sie z. B. in besonderer Schädlichkeit des Tabaksstaubes gesucht wurden, der sogar eine allerdings dem Pathologen unbekannte Tabakosis der Lungen erzeugen sollte, sondern die gleichen auch in anderen Berufsarten 
wirkenden Einflüsse zeigen sich hier in besonderer Steigerung und besonders verhängnisvoller Kombination.

Die Tabaksindustrie steht hinsichtlich ihres Einflusses auf die Art der Tuberkuloseverbreitung in einem gewissen Gegensatz zur Steinhanerei. Während bei letzterer die durch den Steinstaub geschädigten Lungen der Arbeiter der Infektion in Familien und sonstigem Verkehr leichter preisgegeben sind, ist bei der Tabaksindustrie die Infektionsgefahr an der Arbeitsstätte vermehrt: die Arbeitsplätze oft direkt gegenüber mit knappstem Abstand voneinander, - die günstigsten Bedingungen zur Tröpfcheninfektion - der Fussboden mit trockenem pflanzlichem Staub bedeckt, der eine Austrocknung und Verbreitung des Auswurfs der Kranken begünstigt. Dabei sind letztere infolge der geringeren körperlichen Anstrengung, welche die Cigarrenfabrikation erfordert, oft bis in ein weit vorgeschrittenes Stadium ihrer Krankheit befähigt, ihren Beruf auszufüllen.

Interessante Details zu dieser Frage bringen die schon früher erwähnten Berichte der Bezirksärzte an die Regierung. So berechnet Klebe in Bruchsal für die Gesamtbevölkerung eine Mortalität an Tüberkulose von 2,6 auf 1000 Lebende, für die Cigarrenarbeiter von 7,4. Die Zahlen stützen sich allerdings nur auf das eine Jahr 1900, um allzugrossen Anspruch auf Konstanz machen zu können, sind trotzdem jedoch sehr beachtenswert.

In einem den erwähnten Berichten vorangehenden Sammelreferat stellt Hauser (Karlsruhe) für die einzelnen Amtsbezirke die Orte mit Cigarrenindustrie denen ohne dieselbe gegenüber. Er gelangt zu den nebenstehenden tabellarisch geordneten Resultaten, für deren Überlassung ich auch an dieser Stelle meinen besten Dank ausspreche. Die Erhebungen sind für das Jahr 1899 angestellt. Als Massgabe für die Zuverlässigkeit der auf 1000 Lebende bezogenen Mortalitätsziffer und der von mir zugefügten prozentualen Anteilziffern der Tabakarbeiterbevölkerung sind daneben die absoluten Zahlen der Berölkerung gesetzt, auf die sie sich beziehen. Es zeigt sich nun in 15 von 21 Amtsbezirken, in denen sich eine solche Gegenüberstelling ausführen lässt, eine zum Teil recht beträchtliche Steigerung der Tuberkulosemortalität in den Orten mit Tabaksindustrie, in sechs anderen Amtsbezirken findet das Umgekehrte statt. Die Summe der Orte mit Tabakindustrie zeigt eine Mortalität von 2,44 gegen 1,4 der Orte ohne Tabakindustrie. In dieser Zusammenstellung finden sich jedoch auch Amtsbezirke mit inbegriffen, in denen der Prozentsatz der Tabaksarbeiterbevölkerung selbst in den Tabaksindustrieorten weit unter $1 \%$ der Bevölkerung bleibt; dass hier die Unterschiede der Zahlen vielfach auf zufällige Schwan- 
liungen oder andere, wichtigere Einflüsse zurückzuführen sein dürften, bedarf keiner Erörterung. Bis zu einem gewissen Grade vergleichsfähing sind die in der Tabelle durch Fettdruck hervorgehobenen Amtsbezirke, die eine prozentuarische Beteiligung der Tabakarbeiterbevölkerung von über $2 \%$ aufweisen und in denen gleichzeitig die absolute Zahl der Tabakarbeiter eine bedeutendere Höhe zeigt. Bei diesen weisen in bedeutender Mehrheit die Orte mit Tabalssindustrie beträchtlich höhere Mortalitätszahlen auf als die ohne dieselbe. Das umgekehrte Verhältnis zeigen nur Lahr und Sinsheim. Von diesen zeigt Sinsheim so geringe Unterschiede 0,3, dass die Differenz auf einem nur durch Zufall das Jahr betreffenden Verhalten beruhen kann. Als Beispiel hierfür zeigt Weinheim in einem anderen Jahre eine grössere Tuberkulosemortalität in den Orten ohne Tabakindustrie. Ausserdem könnte durch andere Berufe, die sich in den nicht mit Tabaksindustrie beschäftigten Orten finden, eine Erhöhung der Schwindsuchtsmortalität dieser herbeigeführt werden; so z. B. im Bezirk Weinheim durch die Industrie der Holz- and Schnitzstoffe, welche mit einer Verbreitung von über $11 \%$ die häufigste Berufsart darstellt. Eine ganz besondere Stellung nimmt Lahr ein. Dort findet sich die Tabaksindustrie seit langem eingebürgert und hat ihren Sitz in der Stadt Lahr selbst, während sonst die Tabakindustrie gerade die Landbezirke aufgesucht hat. Ausserdem handelt es sich in Lahr fast ausschliesslich um Schnupftabaksfabrikation, für die andere Faktoren massgebend sind als für die Cigarrenfabrikation. Zeigen sich doch auch in dieser verschiedene Verhältnisse der Tuberkulosemorbidität und -Mortalität für die einzelnen Beschäftigungen, so dass die Cigarren- und Wickelmacher den grössten Prozentsatz Phthisiker stellen. Die enorm hohe Sterblichkeitsziffer in den Orten ohne Tabaksindustrie im Amtsbezirke Lahr dürfte sich als eine das Jahr betreffende Zufälligkeit herausstellen, da es sich um eine Promilleberechnung aus nur elf Fällen handelt. Es zeigt sich hier, dass eine Verwertung nur eines Jahrganges schon bedeutende Unsicherheiten in der Aufstellung statistischer Folgerungen herbeiführt. Die Summe dieser Amtsbezirke mit starker Tabakfabrikation zeigt ein Verhältnis der Mortalitätsziffern von 3,0 in den Orten mit zu 1,9 in den Orten ohne Tabakindustrie.

Für diejenigen Amtsbezirke, in denen sich ausser der Cigarrenfabrikation andere industrielle Berufszweige nur wenig verbreitet finden, sondern die übrige Bevölkerung hauptsächlich Ackerbau treibt, wie Emmendingen, Ettenheim, Bruchsal, Schwetzingen zeigt sich diese Vermehrung der Mortalität an Schwindsucht in den Tabaksindustrieorten am reinsten und deutlichsten. Das Gleiche gilt für die Landbezirke von Heidelberg und Mannheim, wo infolge des Übergewichts 
Tabelle über die Mortalität an Schwindsucht für die Amtsbezirke Badens mit bedeutenderer Tabakindustrie (unter teilweiser Benützung einer Tabelle von Hauser mit Ausnahme von Spalte 3, 6 und 7 und der Summenbildung). Trennung der Orte mit und obne Tabakindustrie. Fettgedruckt: Amtsbezirke mit mehr als $2 \%$ Cigarrenarbeiter oder mehr als 1000 in absoluter Zahl.

\begin{tabular}{|c|c|c|c|c|c|c|c|c|c|}
\hline \multirow[b]{2}{*}{$\begin{array}{l}\text { Namen } \\
\text { der Anıtsbezirke }\end{array}$} & \multicolumn{3}{|c|}{ Gesamter Amtsbezirk } & \multicolumn{4}{|c|}{ Orte mit Tabakindustrie } & \multicolumn{2}{|c|}{$\begin{array}{c}\text { Orte ohne } \\
\text { Tabakindustrie }\end{array}$} \\
\hline & 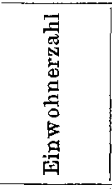 & 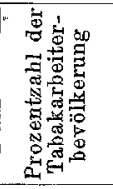 & 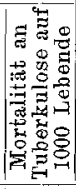 & 茎 & 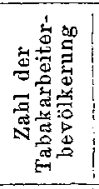 & 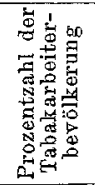 & 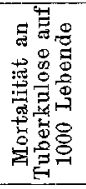 & 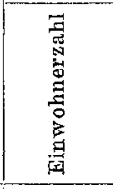 & 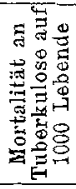 \\
\hline 1 & 2 & 3 & 4 & 5 & 6 & 7 & 8 & 9 & 10 \\
\hline 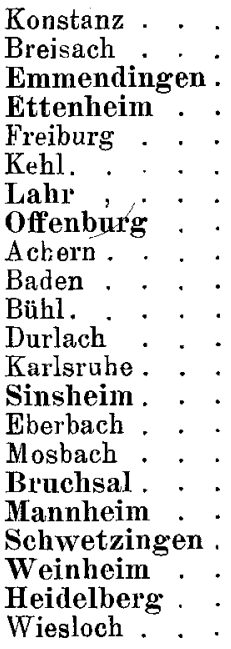 & $\begin{array}{r}47186 \\
19587 \\
\mathbf{4 7 6 9 6} \\
\mathbf{1 7 8 2 6} \\
80517 \\
28450 \\
\mathbf{3 7 6 0 3} \\
\mathbf{5 4 0 9 4} \\
23427 \\
28640 \\
30116 \\
35368 \\
117392 \\
\mathbf{3 4 4 9 2} \\
\mathbf{1 4 7 2 3} \\
30324 \\
\mathbf{6 0 6 6 0} \\
\mathbf{1 2 3 7 3 9} \\
\mathbf{3 2 9 3 3} \\
\mathbf{2 2 6 4 2} \\
\mathbf{8 1 7 2 8} \\
\mathbf{2 2 5 3 6}\end{array}$ & $\begin{array}{c}0,1495 \\
0,388 \\
\mathbf{5 , 5} \\
\mathbf{7 , 9 2} \\
0,2106 \\
1,316 \\
\mathbf{1 0 , 3 2} \\
\mathbf{2 , 2 4} \\
0,3125 \\
0,1566 \\
0,307 \\
\mathbf{1 , 0 1 2} \\
0,297 \\
\mathbf{3 , 6 6} \\
1,126 \\
0,2415 \\
\mathbf{8 , 4 8} \\
\mathbf{2}, \mathbf{1 6} \\
\mathbf{1 7 , 4 8} \\
\mathbf{1 , 4 8 6} \\
\mathbf{5 , 6 0 5} \\
\mathbf{2 4 , 5 5}\end{array}$ & $\begin{array}{l}2,0 \\
1,7 \\
\mathbf{2 , 0} \\
\mathbf{1 , 8} \\
\mathbf{2}, 5 \\
1,5 \\
\mathbf{2 , 3} \\
\mathbf{2 , 7} \\
3,0 \\
2,8 \\
1,6 \\
2,4 \\
2,8 \\
\mathbf{1 , 6} \\
1,6 \\
1,6 \\
\mathbf{3 , 0 3} \\
\mathbf{3 , 0 6} \\
\mathbf{3}, \mathbf{7} \\
\mathbf{2 , 0 3} \\
\mathbf{3 , 4} \\
\mathbf{3 , 4}\end{array}$ & $\begin{array}{r}19974 \\
3365 \\
\mathbf{3 4 9 0 7} \\
\mathbf{1 5 6 1 8} \\
54253 \\
\mathbf{1 3 0 5 2} \\
\mathbf{3 5 0 6 0} \\
\mathbf{4 0 7 2 4} \\
5175 \\
\mathbf{1 8 7 5 8} \\
5498 \\
21771 \\
91484 \\
\mathbf{1 4 9 7 9} \\
5478 \\
1064 \\
\mathbf{5 0 5 3 6} \\
\mathbf{1 2 0 9 7 6} \\
\mathbf{3 1 4 9 9} \\
\mathbf{6 0 8 2} \\
\mathbf{6 2 9 5 6} \\
22536\end{array}$ & $\begin{array}{r}70 \\
77 \\
\mathbf{2 6 6 5} \\
\mathbf{1 4 0 8} \\
168 \\
369 \\
\mathbf{3 8 4 5} \\
\mathbf{1 1 9 7} \\
72 \\
49 \\
91 \\
350 \\
\mathbf{5 6 1} \\
\mathbf{1 2 7 2} \\
169 \\
74 \\
\mathbf{5 0 0 6} \\
\mathbf{2 6 2 3} \\
\mathbf{5 7 1 8} \\
\mathbf{3 9 5} \\
\mathbf{4 5 7 8} \\
5524\end{array}$ & $\begin{array}{c}0,35 \\
2,28 \\
\mathbf{7 , 6 3} \\
\mathbf{9 , 0 6} \\
0,31 \\
2,74 \\
\mathbf{1 0 , 9 5} \\
\mathbf{2 , 9 4} \\
\mathbf{1 , 3 9} \\
0,26 \\
\mathbf{1}, 65 \\
\mathbf{1}, 6 \\
0,395 \\
\mathbf{8 , 4 8} \\
3,1 \\
\mathbf{6 , 9 5} \\
\mathbf{9 , 5 6} \\
\mathbf{2 , 1 6} \\
\mathbf{1 8 , 1} \\
\mathbf{5 , 5} \\
\mathbf{7 , 2 6} \\
\mathbf{2 4 , 5 5}\end{array}$ & 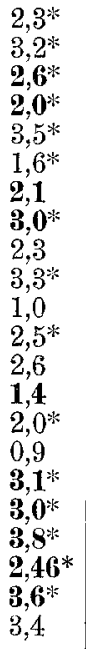 & $\begin{array}{r}27213 \\
16222 \\
\mathbf{1 2 7 8 9} \\
\mathbf{2 2 0 8} \\
26264 \\
15398 \\
\mathbf{2 5 4 3} \\
\mathbf{1 3 3 7 0} \\
18252 \\
9882 \\
24618 \\
13597 \\
25908 \\
\mathbf{1 9 5 1 3} \\
9245 \\
29260 \\
\mathbf{1 0 1 2 4} \\
\mathbf{2 7 6 3} \\
\mathbf{1 4 3 4} \\
\mathbf{1 6 5 6 0} \\
\mathbf{1 8 7 7 2} \\
\mathbf{-}\end{array}$ & 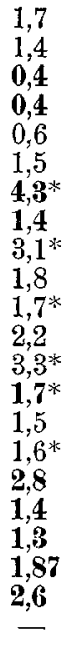 \\
\hline $\begin{array}{l}\text { Gesamtsumme } \\
\text { Summe der fett- } \\
\text { gedruekten Amts- } \\
\text { bezirke . . . }\end{array}$ & $\begin{array}{l}991679 \\
\mathbf{5 1 3 4 1 3}\end{array}$ & 3,63 & 2,79 & $\begin{array}{l}675745 \\
\mathbf{4 1 3 3 3 7}\end{array}$ & $\begin{array}{l}36081 \\
28689\end{array} \mid$ & 5,33 & 3,00 & $\begin{array}{l}315934 \\
\mathbf{1 0 0 0 7 6}\end{array}$ & $\begin{array}{l}1,9 \\
1,9\end{array}$ \\
\hline
\end{tabular}

der grossen Städte mit beruflich gemischter Bevölkerung für den ganzen Bezirk die Zahlen weniger deutlich zum Ausdruck kommen. Es zeigt sich auch hier wieder das Erfordernis detaillierter, für den bestimmten Zweck erhobener Einzelerhebungen an kleineren Objekten nach einbeitlichem Schema und erst sekundärer Zusammensetzung zu umfassenderer Statistik. Es ist dies ein Erfordernis, um das wir nicht herumkommen, soll überhaupt der Versuch gemacht werden, auf statistischem Wege der Verbreitungsart und den Verbreitungsbedingungen der Tuberkulose näher zu kommen. 
Ähnliche Betrachtungen, wie sie für den Einfluss der Tabaksindustrie auf die Tuberkuloseverbreitung angestellt wurden, haben auch für die anderen Berufszweige ihre Gültigkeit. Es ist überall die Wechselwirkung zwischen Schädigung der Atemorgane durch und während der Arbeit, Infektionsgefahr auf dem Arbeitsplatz und den aus der Zusammensetzung der betreffenden Bevölkerung resultierenden Faktoren: Anzahl der Erwerbstätigen - Männer und Frauen und nicht im Beruf thätigen Angehörigen, und Prozentverhältnis der gesamten Berufsgruppe zur Bevölkerung, welche uns Rückschlüsse auf die Tuberkulosenverbreitung in der betreffenden Gegend gestatten. Welche anderen Faktoren sonst noch im Spiele sind, darüber konnte die vorläufige Untersuchung noch nicht überall den gewünschten Aufschluss geben. Es bleibt der auf der Einzelbeobachtung aufgebauten Statistik vielleicht und hoffentlich vorbehalten, hierin manches Licht zu verbreiten.

\section{Disposition.}

Ein Punkt, der zur Pathogenese der Schwindsucht in innigste Beziehungen gebracht wird, hat bisher noch keine Erwähnung gefunden. Es ist dies die Disposition. Es sei mir erlaubt, die umfangreiche, widerspruchsvolle Literatur über diesen Gegenstand zu übergehen, $\mathrm{da}$ dieselbe in späteren Ausführungen eine ausgedehnte Besprechung finden soll. Nur soviel sei hier hervorgehoben, dass unter Disposition von den verschiedenen Autoren das Verschiedenste verstanden wird, das nur das einzig Gemeinsame hat, Ausdruck einer verminderten Widerstandskraft des Individuums gegen die Tuberkulose zu sein. Fassen wir daher vorläufig den Ausdruck Disposition als einen derartigen Sammelbegriff auf, ohne ihm eine ins Detail gehende Bedeutung beizulegen.

Nehmen wir nun mit der Mehrzahl der Ärzte das Bestehen einer solchen individuell wechselnden Disposition an, so kann sie wohl, wie schon früher bemerkt, für das einzelne Individuum von grösster Bedeutung sein, ob es Tuberkulose acquiriert oder nicht, für die grosse Masse kommt sie statistisch nicht zum Ausdruck, da diese gleichmässig aus Disponierten und Nichtdisponierten zusammengesetzt ist. Nicht die Zahl der Erkrankungen kann also durch die Disposition beeinflusst werden, sondern nur die Auswahl der erkrankenden Individuen.

Erst wenn bei einem Vergleich zweier Bevölkerungsgruppen, z. B. zweier Bezirke mit gleichen Infektionsgelegenheiten und Häufigkeit derselben sich verschiedene Erkrankungsziffern finden, kann man an 
eine für die beiden Bezirke verschiedene Zusammensetzung aus Disponierten und Nichtdisponierten denken. Die Art der Disposition, welche also bei dem von uns gebrauchten Einteilungsmodus in Betracht käme, wäre die Rassendisposition. Anhaltspunkte für das Bestehen desselben finden sich in der verschiedenen Tuberkulosehäufigkeit verschiedener Völker, die sich z. B. auch in den verschiedenen Krankheitsziffern der Soldaten aus verschiedenen Volksstämmen der östreichisch-ungarischen Monarchie zeigt.

Es fragt sich nun, ob sich aus den für Baden aufgestellten Zahlen ähnliche Wahrnehmungen gewinnen lassen. Baden ist der Hauptsache nach von zwei Volksstämmen bewobnt, dem alemannischen im Süden und einem hauptsächlich fränkischen Mischstamm im Norden. Die Grenze beider stellt etwa die Kinziglinie dar in der Weise, dass die nach Norden sich öffnenden Längstäler des Schwarzwalds noch der die Alemannen zurückdrängenden fränkischen Bevölkerung als Eingangspforte dienten.

Nun fällt allerdings ein gewisser Gegensatz in der Tuberkuloseverbreitung im Norden und Süden sofort in die Augen. Aber wir haben gesehen, dass sich die meisten der die Verbreitung der Tuberkulose begünstigenden Momente auch im Norden häufiger finden, als im Süden. In allen Fällen scheinen diese jedoch nicht zur Erklärung des Gegensatzes auszureichen, sondern noch eine Lücke zu lassen, für deren Ausfüllung sich vielleicht in der Heranziehung einer solchen Rassendisposition ein Mittel finden liesse.

Nehmen wir als erstes Beispiel den Gegensatz der grossen Tabaksindustriegebiete im Norden und Süden. Die äusseren Verhältnisse sind sich sehr ähnlich, es zeigen sich nur geringe Verschiedenheiten in Höhe, Klima, Bodenbeschaffenheit. Der Unterschied in der Zusammensetzung der Bevölkerung aus Ackerkau- und Industrietreibenden lässt zwar einen Vorteil zu Gunsten des Südens erkennen, das Verhältnis ist dort etwa 1:1, während im Norden die Industrie die Landwirtschaft überwiegt. Auch der spezielle Anteil der Tabaksfabrikation erreicht im Süden kaum 10\%, während er im Norden durchweg darüber, vereinzelt sogar über $20 \%$ beträgt. Der Unterschied in der Tuberkulosemortalität ist jedoch so beträchtlich, dass man diese Momente nicht gut allein dafür verantwortlich machen kann, sondern dass es gerechtfertigt erscheint, hier Einflüsse der Rassendisposition in Diskussion zu ziehen.

Noch deutlicher zeigt sich der Unterschied in den nicht mit Tabakfabrikation beschäftigten Orten beider Komplexe, wo also die erwähnten anderen Einflüsse sich nicht geltend machen können. Dies sind hier wie dort fast reine Ackerbauorte. Und doch ein gewal- 
tiger Unterschied in der Tuberkulosemortalität $0,4 \%$ im Süden 2$3 \%$ und darüber im Norden.

Im gleichen Sinne spricht der geringe sich bemerkbar machende Einfluss der Textilindustrie, die hauptsächlich in den südlichen Bezirken ihren Sitz hat, im gleichen Sinne der Gegensatz in der Schwindsuchtsterblichkeit zwischen den südlichen Bezirken mit starker Industrie der Holz- und Schnitzstoffe, insbesondere Bürstenfabrikation und dem nördlichen Weinheim. Es sind dies Fragen zu deren Aufstellung uns dieses Übersichtsbild Anlass gibt, deren Beantwortung aber erst nach eingehenderen Detailstudien möglich sein wird. Vorlänfig muss in der Dipositionsfrage auf Grund der vorliegenden Zahlen und Erörterungen dahin entschieden werden: auf statistischem Wege kann kein Nachweis geliefert werden, dass sie nicht bestehen kan $n$; ob sie jedoch der richtige Schlussstein ist, um gewisse noch offene Lücken in Ringe der Beweisführung zu schliessen, darauf kann als Antwort einstweilen nur ein non liquet erfolgen.

\section{Schlusssätze.}

Fassen wir die Hauptgesichtspunkte und Ergebnisse der vorliegenden Arbeit noch einmal kurz zusammen, so zeigt sich für das Grossherzogtum Baden ein Zusammenhang der geographischen Verbreitung der Tuberkulose mit folgenden Verhältnissen:

Mit zunehmender Erhebung über den Meeresspiegel, sinkt die Tuberkulosemortalität der Bewohner. Dieses Absinken wird gesteigert.

1. Durch den häufigeren Betrieb der Landwirtschaft in grösserer Höhe,

2. vielleicht durch geringere Volksdichte,

3. durch im einzelnen nicht eliminierbare Faktoren, die mit dem geographischen Höhenbegriff in direkterem Zusammenhang stehen, über deren Art aber noch zu wenig bekannt ist.

Für den Einfluss bestimmter Berufsarten auf die Schwindsuchtsverbreitung innerhalb der ganzen Bevölkerung kommt in Betracht:

1. ihre prozentuarische Beteiligung an der Zusammensetzung der Bevölkerung.

2. Die Zusammensetzung der betreffenden Berufsart aus Erwerbstätigen, - wobei eine ausgedehnte Erwerbstätigkeit der Frauen im allgemeinen einen Nachteil bedeutet, - und nicht in Beruf beschäftigten Angehörigen. 
3. Schädigung durch den Beruf oder vermehrte Infektionsgefahr an dem Arbeitsplatz.

Im allgemeinen zeigt sich $Z$ unahme der Tuberkulosemortalität mit Zunahme der Industrie und Abnahme der Landwirtschaft.

Kein Einfluss konnte auf statistischem Wege nachgewiesen werden für Armut, Ernährungsweise, Alkoholkonsum. Doch ist hier noch eine Kontrolle der verwandten Zahlen im Detail abzuwarten.

Ein Gegensatz in der geographischen Verbreitung besteht zwischen Krebs und Tuberkulose, indem sich letztere mehr im Norden zu hohen Mortalitätsziffern aufschwingt, während der Krebs im Süden bedeutendere Zahlen erreicht.

Ein Einfluss einer Rassendisposition ist wahrscheinlich, doch exakt einstweilen noch nicht nachzuweisen.

Zum Schlusse meiner Arbeit ist es mir eine angenehme Pflicht, Herrn Geheimen Hofrat Dr. Knauf und Herrn Professor Brauer meinen Dank auszusprechen für die mannigfachen Anregungen, die zur Formulierung der Fragestellung führten, insbesondere aber letzterem zu danken für freundliche Unterstützung bei der Abfassung der Arbeit.

\section{Literaturverzeichnis.}

1. A ufrecht, Zur Verhütung und Heilung der Lungentuberkulose. Wien 1898.

2. Brauer, Die Verbreitung der Tuberkulose in Tabakfabriken. Bericht über den Kongress zum Bekämpfen der Tuberkulose als Volkskrankheit. 1899.

3. Brehmer, Die Gesetze und Heilbarkeit der chronischen Lungentuberkulose. Berlin 1856.

4. Cornet, Die Tuberkulose. Notnagels Handbuchs. Wien 1900.

5. Derselbe, Über Tuberkulose. Leipzig 1890.

6. Derselbe, Über einige der nächsten Ziele der T'uberkulosenforschung. Zeitschr. f. Tbe. u. Hstw. Bd. V. p. 48.

7. Köhler, Allgemeines über die Ausbreitung und Bedeutung der Tuberkulose als Volkskrankheit. Kongressbericht 1899.

8. Krieger, Beziehungen zwischen den äusseren Lebensverhältnissen und der Ausbreitung der Tuberkulose. Kongressbericht 99.

9. Kuthy, Die Ausbreitung der Lungenschwindsucht in Ungarn. Kongressbericht 1899. 
92 W. Hoffmann. Beitrag zur Kenntnis der Tuberkuloseverbreitung in Baden. [44

10. Lubarsch-Ostertag, Lubarsch, Infektionswege und Krankheitsdisposition. Ergebnisse der allgemeinen Pathologie und pathol. Anatomie.

11. Martins, Pathogenese innerer Krankheiten. Leipzig u. Wien. Fr. Denticke. 1900.

12. Meyer, Über das Vorkommen von Tuberknlose bei den Berliner Buchdruckern und Schriftsetzern. Kongressbericht 1899.

13. Moritz, Zur Verbreitung der Tuberkulose unter den mit Staubentwickelung verbundenen Berufsarten. Kongressbericht 1899.

14. Naus, Konstitutionspathologie. Pathologie der Zukunft. München 1901.

15. Neumann, Die Veränderungen der Volksdichte im südlichen Schwarzwald 1852-1895. Freiburg i. B. 1896.

16. Ransome. The Constitution of infection by Tubercle. Zeitschr. f. Tuberk. u. Heilstättenwesen. Bd. I. p. 7.

17. Reibmeier, Die Ehe Tuberkulöser und ihre Folgen. Leipzig und Wien 1894.

18. Derselbe, Inzucht und Vermischung beim Menschen. Leipz. u. Wien 1897.

19. Riffel, Erblichkeit der Schwindsucht und tuberkulösen Prozesse. Karlsruhe 1891.

20. Derselbe, Mitteilungen über Erblichkeit und Infektiosität der Schwindsucht. Leipzig 1892.

21. Derselbe, Weitere pathologische Studien über Schwindsucht und Krebs und einige andere Krankheiten.

22. Rubner, Lehrbuch der Hygiene. p. $886 \mathrm{ff}$.

23. Schmid, Die Verbreitung der Tuberkulose in der Schweiz. Kongress zur Bekämpfung der Tuberkulose. 1899.

24. Statistisches Jabrbuch für Baden. 1891-1900.

25. Statistische Mitteilungen für Baden. 1891-1900.

26. Stralmann, Die Tuberkulose unter den Stahlschleifern. Kongressbericht 93.

27. Topographische Karte für das Grossherzogtum Baden.

28. Uhlig, Die Veränderungen der Volksdichte im nördlichen Baden 18521895. Stuttgart 1898.

29. Virchow, Virchows Archiv für pathologische Anatomie. Bd. II. p. 170.

30. Wörrishofer, Die soziale Lage der Cigarrenarbeiter im Grossherzogtum Baden. Karlsruhe 1890.

31. Derselbe, Die soziale Lage der Fabrikarbeiter in Mannheim. Karlsruhe 1891.

32. Derselbe, Über den Einfluss sozialer Verhältnisse auf die Hänfigkeit der Schwindsucbtstodesfälle. Kongressbericht 1899.

33. Würzburg, Über den Einfluss des Alter's und Geschlechts auf die Sterblichkeit an Lungenschwindsucht. Mitteilungen aus dem Kaiserlichen Gesundheitsamte. 2. 1884. 